\title{
Optical and near-infrared follow-up observations of four Fermi/LAT GRBs: redshifts, afterglows, energetics, and host galaxies ${ }^{\star}$
}

\author{
S. McBreen ${ }^{1,2}$, T. Krühler ${ }^{2,3}$, A. Rau ${ }^{2}$, J. Greiner ${ }^{2}$, D. A. Kann ${ }^{4}$, S. Savaglio ${ }^{2}$, P. Afonso ${ }^{2}$, C. Clemens ${ }^{2}$, R. Filgas ${ }^{2}$, \\ S. Klose ${ }^{4}$, A. Küpcü Yoldaş ${ }^{5}$, F. Olivares E. ${ }^{2}$, A. Rossi ${ }^{4}$, G. P. Szokoly ${ }^{6}$, A. Updike ${ }^{7}$, and A. Yoldaş ${ }^{2}$ \\ 1 School of Physics, University College Dublin, Dublin 4, Ireland \\ e-mail: Sheila.McBreen@ucd.ie \\ 2 Max-Planck-Institut für extraterrestrische Physik, 85748 Garching, Germany \\ 3 Universe Cluster, Technische Universität München, Boltzmannstrasse 2, 85748 Garching, Germany \\ 4 Thüringer Landessternwarte Tautenburg, Sternwarte 5, 07778 Tautenburg, Germany \\ 5 European Southern Observatory, 85748 Garching, Germany \\ 6 Institute of Physics, Eötvös University, Pázmány P. s. 1/A, 1117 Budapest, Hungary \\ 7 Department of Physics and Astronomy, Clemson University, Clemson, SC 29634, USA
}

Received 24 November 2009 / Accepted 16 March 2010

ABSTRACT

\begin{abstract}
Aims. Fermi can measure the spectral properties of gamma-ray bursts over a very large energy range and is opening a new window on the prompt emission of these energetic events. Localizations by the instruments on Fermi in combination with follow-up by Swift provide accurate positions for observations at longer wavelengths leading to the determination of redshifts, the true energy budget, host galaxy properties and facilitate comparison with pre-Fermi bursts.

Methods. Multi-wavelength follow-up observations were performed on the afterglows of four bursts with high energy emission detected by Fermi/LAT: GRB 090323, GRB 090328, GRB 090510 and GRB $090902 B$. They were obtained in the optical/near-infrared bands with GROND mounted at the MPG/ESO $2.2 \mathrm{~m}$ telescope and additionally of GRB 090323 in the optical with the $2 \mathrm{~m}$ telescope in Tautenburg, Germany. Three of the events are classified as long bursts while GRB 090510 is a well localized short GRB with $\mathrm{GeV}$ emission. In addition, host galaxies were detected for three of the four bursts. Spectroscopic follow-up was initiated with the VLT for GRB 090328 and GRB 090510.

Results. The afterglow observations in 7 bands are presented for all bursts and their host galaxies are investigated. Knowledge of the distance and the local dust extinction enables comparison of the afterglows of LAT-detected GRBs with the general sample. The spectroscopic redshifts of GRB 090328 and GRB 090510 were determined to be $z=0.7354 \pm 0.0003$ and $z=0.903 \pm 0.001$ and dust corrected star-formation rates of $4.8 M_{\odot} \mathrm{yr}^{-1}$ and $0.60 M_{\odot} \mathrm{yr}^{-1}$ were derived for their host galaxies, respectively.

Conclusions. The afterglows of long bursts exhibit power-law decay indices $(\alpha)$ from less than 1 to $\sim 2.3$ and spectral indices $\left(\beta_{\text {opt }}\right)$ values from 0.65 to $\sim 1.2$ which are fairly standard for GRB afterglows. Constraints are placed on the jet half opening angles of $\lesssim 2.1^{\circ}$ to $\gtrsim 6.4^{\circ}$, which allows limits to be placed on the beaming corrected energies. These range from $\lesssim 5 \times 10^{50}$ erg to the one of the highest values ever recorded, $\gtrsim 2.2 \times 10^{52} \mathrm{erg}$ for GRB 090902B, and are not consistent with a standard candle. The extremely energetic long Fermi bursts have optical afterglows which lie in the top half of the brightness distribution of all optical afterglows detected in the Swift era or even in the top 5\% if incompleteness is considered. The properties of the host galaxies of these LAT detected bursts in terms of extinction, star formation rates and masses do not appear to differ from previous samples.
\end{abstract}

Key words. gamma-ray burst: individual: GRB 090323 - gamma-ray burst: individual: GRB 090328 gamma-ray burst: individual: GRB 090510 - gamma-ray burst: individual: GRB 090902B

\section{Introduction}

The follow-up of gamma-ray bursts (GRBs) detected by the Swift satellite (Gehrels et al. 2004) has led to the determination of the distance scale for a large sample of bursts. The Burst Alert Telescope (BAT, Barthelmy et al. 2005) is sensitive in the energy range $15-150 \mathrm{keV}$ and has good localization capabilities with typical uncertainties in the arcminute range. Rapid follow-up by

* Based on observations made with the ESO Telescopes at the La Silla Paranal Observatories under programme ID 083.D-0903 and 283.D-5059, the MPG/ESO $2.2 \mathrm{~m}$ Telescope at La Silla Observatory and the Schmidt telescope of the Thüringer Landessternwarte Tautenburg.
Swift's narrow field instruments in the X-rays (XRT, Burrows et al. 2005) and optical/UV (UVOT, Roming et al. 2005) have lead to the arcsecond localizations required for ground-based observers and in turn to spectroscopic redshift measurements of a large sample of GRBs (e.g., Fynbo et al. 2009) and investigation of their host galaxies (e.g., Savaglio et al. 2009; Perley et al. 2009 b). To date distances to $\sim 200$ GRB sources have been established with redshifts ranging from $z=0.0085$ (GRB 980425: Tinney et al. 1998; Galama et al. 1998) to $z \sim 8.2$ (GRB090423: Tanvir et al. 2009; Salvaterra et al. 2009). The BAT has a narrow spectral range and is not able to determine the spectral parameters of the prompt emission of GRBs over a broad energy range. Since the launch of the Fermi Gamma-ray Space 
Telescope (Atwood et al. 1994; Michelson 1996) there is now the possibility to investigate the spectrum over seven decades in energy. These events can be localized by instruments from Fermi and followed up by Swift and ground-based obervatories, enabling investigation of their afterglows and host properties and facilitating comparison to a general sample. In some fortuitous cases instruments on the Fermi and Swift satellites may trigger on the same event with high-energy emission enabling both rapid follow-up and broadband prompt emission coverage (e.g. GRB 090510, see Sect. 2.3). The high-energy spectral properties of GRBs were investigated previously by instruments onboard the Compton Gamma-ray Observatory, however the redshifts of these events are unknown. The spectra of these GRBs were described by an extrapolation of the low energy spectra to energies $>100 \mathrm{MeV}$ (e.g., Hanlon et al. 1994), and in some cases an additional component at high-energies (González et al. 2003; Kaneko et al. 2008) and long-lived GeV emission (Hurley et al. 1995). Recently a photometric redshift of $z=1.8_{-0.3}^{+0.4}$ was reported by Rossi et al. (2008) for GRB 080514B which was detected at energies up to $300 \mathrm{MeV}$ by instruments on the AGILE satellite (Giuliani et al. 2008).

Fermi hosts two instruments that detect GRBs: the Gamma-ray Burst Monitor (GBM: Meegan et al. 2009) sensitive to photons from $\sim 8 \mathrm{keV}$ to $\sim 40 \mathrm{MeV}$, and the Large Area Telescope with a spectral coverage from $\sim 30 \mathrm{MeV}$ to $\sim 100 \mathrm{GeV}$ (LAT: Atwood et al. 2009). Together they provide valuable information on the spectral properties of the prompt emission (e.g. Abdo et al. 2009a,d,b,c), the presence or absence of intrinsic spectral cut-offs or those due to the optical depth of the universe to high-energy $\gamma$-rays due to pair production on infrared diffuse Extragalactic Background Light (e.g., de Jager \& Stecker 2002; Metcalfe et al. 2003; Kneiske et al. 2004; Kashlinsky et al. 2005; Stecker et al. 2006; Franceschini et al. 2008; Finke et al. 2010; Abdo et al. 2009b) and test for quantum gravity effects (e.g., Amelino-Camelia et al. 1998; Scargle et al. 2008; Mattingly 2005; Amelino-Camelia \& Smolin 2009; Abdo et al. 2009c,a).

Up to the end of January 2010, fourteen GRBs have been detected by both instruments and localized by the LAT: GRB 080825C (Bouvier et al. 2008; Abdo et al. 2009d), GRB 080916C (Abdo et al. 2009c), GRB 081024B (Omodei 2008), GRB 081214 (Wilson-Hodge et al. 2008), GRB 090217 (Ohno et al. 2008), GRB 090323 (Ohno et al. 2009), GRB 090328 (McEnery et al. 2009b), GRB 090510 (Ohno \& Pelassa 2009; Abdo et al. 2009a), GRB 090626 (Piron et al. 2009), GRB 090902B (de Palma et al. 2009b; Abdo et al. 2009b), GRB 090926A (Bissaldi 2009; Uehara et al. 2009), GRB 091003 (McEnery et al. 2009a), GRB 091031 (Palma et al. 2009), and GRB 100116A (McEnery et al. 2010). Twelve are long duration GRBs and two have reported durations compatible with the short burst class (GRBs 081024B and 090510). The redshifts of five of these bursts have been determined and range from $z=0.736$ to $z=4.35 \pm 0.15$ (Greiner et al. 2009a; Updike et al. 2009b; Chornock et al. 2009; Cenko et al. 2009; Rau et al. 2009b; Cucchiara et al. 2009; Malesani et al. 2009).

Among the most impressive of these events are GRB 080916C (Abdo et al. 2009c) and GRB 090510 (Abdo et al. 2009a). GRB 080916C is a long bright GRB for which the prompt emission spectrum could be fit over six decades in energy by the empirical Band function (see Abdo et al. 2009 c, for detailed results). The burst was found to have a high photometric redshift of $z=4.35 \pm 0.15$ via ground-based follow up observations (Greiner et al. 2009a). The distance information enabled the determination of the rest frame properties, energetics, and the placing of lower limits on the bulk Lorentz factor of the outflow ( $\gtrsim 900-1100$ ) (Abdo et al. 2009c; Greiner et al. 2009a). GRB 090510 is a short burst with a $T_{90}$ of $2.1 \mathrm{~s}$ and from which a $31 \mathrm{GeV}$ photon was detected (Abdo et al. 2009a). The distance determination (Rau et al. 2009b) and high energy emission allow a limit to be placed on photon dispersion (Abdo et al. 2009a). Moreover, very recently a $33 \mathrm{GeV}$ photon was detected from GRB 090902B (Abdo et al. 2009b) and this burst is at redshift $z=1.822$ (Cucchiara et al. 2009). Clearly, the distance determination via optical spectroscopy is crucial to the interpretation of these events.

We report on the optical and near-infrared (NIR) observations of the afterglow of four bursts with high energy emission, GRB 090323, GRB 090328, GRB 090510 and GRB 090902B and compare them to the sample of GRB afterglows to date. Furthermore we also report on the spectroscopic redshift determination for GRB 090328 and GRB 090510. Throughout the paper, we adopt concordance $\Lambda$ CDM cosmology $\left(\Omega_{\mathrm{M}}=0.27\right.$, $\Omega_{\Lambda}=0.73, H_{0}=71 \mathrm{~km} \mathrm{~s}^{-1} \mathrm{Mpc}^{-1}$ ), and the convention that the flux density of the GRB afterglow can be described as $F_{v}(t) \propto v^{-\beta} t^{-\alpha}$

\section{Observations}

\subsection{GRB 090323}

At 00:02:42.63 UT on 23 March 2009, Fermi GBM triggered and located the long burst GRB 090323 (Ohno et al. 2009). An excess of $5 \sigma$ was detected by the LAT and the burst was localized to $\mathrm{RA}(\mathrm{J} 2000), \operatorname{dec}(\mathrm{J} 2000)=190.69,+17.08$ degrees with a $68 \%$ containment radius of $0.09^{\circ}$. Furthermore, it was reported that emission continued up to a few kiloseconds post trigger (Ohno et al. 2009). A triangulation by the Inter Planetary Network localized the burst to an arc which intersected the GBM and LAT positions (Hurley et al. 2009). Swift carried out a Target of Opportunity observation $\sim 19 \mathrm{~h}$ post burst and a fading afterglow was found (Kennea et al. 2009a; Perri \& Stratta 2009). Updike et al. (2009b) observed the position of the X-ray afterglow with the Gamma-ray Burst Optical/Near-infrared Detector (GROND) in 7 bands $\sim 27 \mathrm{~h}$ after the burst and detected a source for which they reported a preliminary photometric redshift of $4.0 \pm 0.3$ (see below). Chornock et al. (2009) reported a spectroscopic redshift of $z=3.57$ based on observations of the optical afterglow using the Gemini Multi-Object Spectrograph (GMOS) mounted on the Gemini South Telescope. The GRB was also detected in the radio band (Harrison et al. 2009; van der Horst 2009).

Using the reported spectral parameters (Bissaldi et al. 2010) and a redshift of $z=3.57$ (Chornock et al. 2009) $E_{\gamma \text {,iso is }}$ $4.1 \times 10^{54} \mathrm{erg}$ in the $1 \mathrm{keV}$ to $10 \mathrm{MeV}$ range, or $5.1 \times 10^{54} \mathrm{erg}$ in the $1 \mathrm{keV}$ to $10 \mathrm{GeV}$ range with a restframe peak energy of $2.7 \pm 0.2 \mathrm{MeV}$. The $E_{\gamma \text {,iso }}$ is one of the highest ever measured even rivals GRB 080916C for the most energetic GRB.

The field of GRB 090323 was observed simultaneously in 7 bands $\left(g^{\prime} r^{\prime} i^{\prime} z^{\prime} J H K_{\mathrm{S}}\right)$ by GROND, mounted at the $2.2 \mathrm{~m}$ MPG/ESO telescope at La Silla Observatory, Chile (Greiner et al. 2008) and using the Schmidt-camera at the AlfredJensch telescope of the Thüringer Landessternwarte Tautenburg (TLS), Germany. GROND and TLS observations started on 24th March 2009, $27 \mathrm{~h}$ and $45 \mathrm{~h}$ after the GBM trigger, respectively (Updike et al. 2009b; Kann et al. 2009c). Observations continued in the upcoming nights (Kann et al. 2009d,b) and the last GROND epoch was obtained $\sim 100$ days after $T_{0}$. The best optical position of the afterglow measured in the $r^{\prime}$ band against the SDSS catalogue is $\mathrm{RA}(\mathrm{J} 2000)=12: 42: 50.28$, 


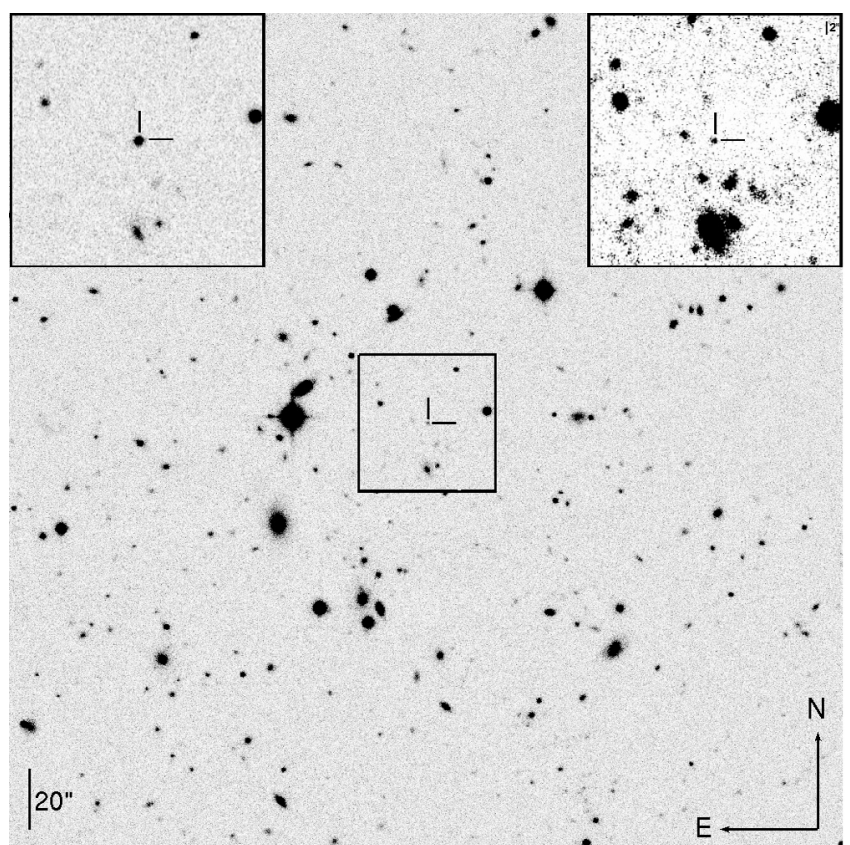

Fig. 1. GROND finding chart for the afterglow of GRB 090323. The main panel shows the field of GRB 090323 taken 3.5 days post burst. The field shown has $4^{\prime} \times 4^{\prime}$, where North is up and East is to the left. The afterglow position is indicated with two lines in each image. The inset in the top left shows a zoom into the afterglow region at the earliest epoch, where the afterglow was still bright. All images are $i^{\prime}$ band, except the host image ( $r^{\prime}$, top right inset), which is also enhanced in contrast for demonstration purposes.

$\operatorname{dec}(\mathrm{J} 2000)=+17: 03: 11.9$ with systematic and statistical uncertainties of 0.1 in each coordinate. A finding chart of the afterglow is shown in Fig. 1.

\subsection{GRB 090328}

At 09:36:46 UT on 28 March 2009, Fermi GBM triggered and located a long burst GRB 090328. The event was significantly detected up to a few $\mathrm{GeV}$ at the $5 \sigma$ level by the Fermi/LAT and was localized to $\mathrm{RA}(\mathrm{J} 2000), \operatorname{dec}(\mathrm{J} 2000)=90.87,-41.95$ with a $68 \%$ containment radius of $0.11 \mathrm{deg}$ (McEnery et al. $2009 \mathrm{~b})$. The emission in the LAT was reported to last until about 900 s post trigger by Cutini et al. (2009). A Swift Target of Opportunity observation was initiated and XRT observations commenced $\sim 16 \mathrm{~h}$ post burst (Kennea 2009) and X-ray and bright UV/optical afterglow candidates were subsequently reported by Kennea et al. (2009b) and Oates (2009). A spectrum of the optical afterglow was taken with the GMOS instrument mounted on the Gemini South Telescope (Cenko et al. 2009) and the redshift was reported to be $z=0.736$. Further photometric observations of the afterglow were reported in the optical (Allen et al. 2009) and optical/NIR with GROND (Updike et al. 2009a, see below) and additionally in the radio band (Frail et al. 2009).

The time averaged spectrum $\left(T_{0}+3.1\right.$ to $\left.T_{0}+29.7 \mathrm{~s}\right)$ of the prompt emission was reported by GBM to be best fit by a Band function with indices $\alpha=-0.93 \pm 0.02$ and $\beta=-2.2 \pm 0.1$, a peak energy of $E_{\text {peak }}=653 \pm 45 \mathrm{keV}$ with an event fluence of $(9.5 \pm 1.0) \times 10^{-5} \mathrm{erg} / \mathrm{cm}^{2}$ in the $8 \mathrm{keV}$ to $40 \mathrm{MeV}$ band (Rau et al. 2009a). Bissaldi et al. (2010) report that the spectrum $\left(T_{0}+0\right.$ to $\left.T_{0}+66.6 \mathrm{~s}\right)$ is best fit by a power-law with exponential cut off. The index of the power-law is $-1.07 \pm 0.02$ and the peak energy is $744_{-47}^{+50} \mathrm{keV}$. At a redshift of 0.736 , these

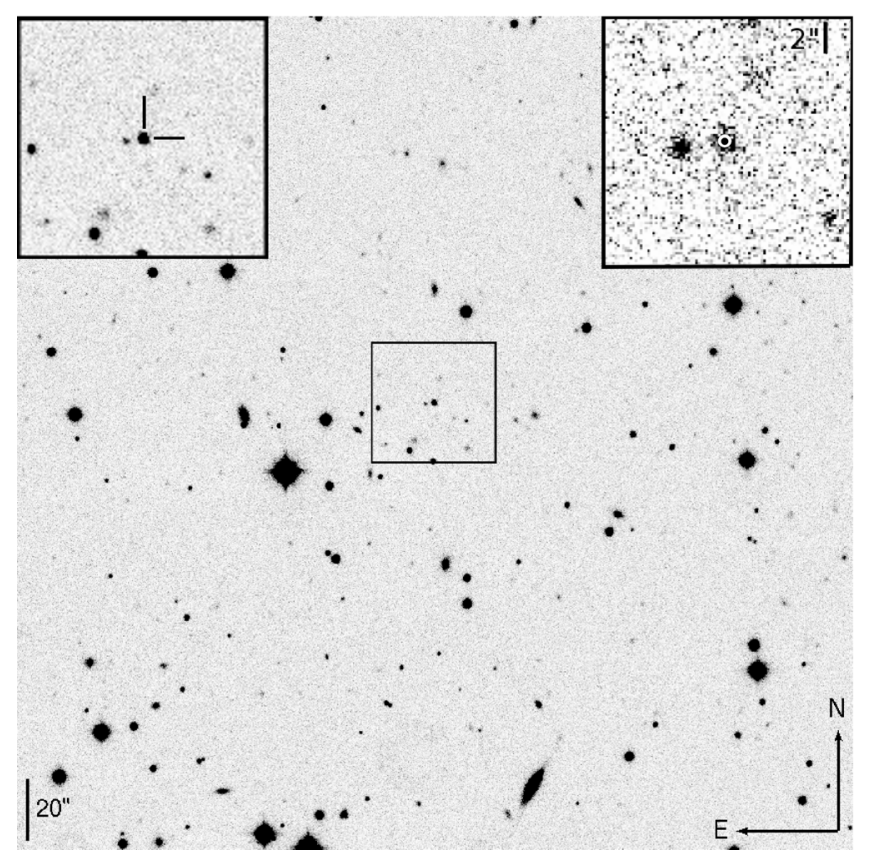

Fig. 2. GROND finding chart for the afterglow of GRB 090328. The main panel shows the field of GRB 090328 taken 1.5 days post burst. The field shown has $4^{\prime} \times 4^{\prime}$, where North is up and East is to the left. The inset in the top left shows a zoom into the afterglow region at the same epoch, where the afterglow is indicated with two lines. The inset on the top right contains a $15^{\prime \prime} \times 15^{\prime \prime}$ region and shows a host image taken 10 days post burst. The afterglow position is shown by a small white circle of radius 0.3 . All images are in the $i^{\prime}$ band.

spectral parameters correspond to $E_{\gamma, \text { iso }}$ in the $1 \mathrm{keV}$ to $10 \mathrm{MeV}$ range of $1.0 \times 10^{53} \mathrm{erg}$ and $1.0 \times 10^{53} \mathrm{erg}$ in the $1 \mathrm{keV}$ to $10 \mathrm{GeV}$ band with a restframe peak energy of $E_{\text {peak }}^{\text {rest }}=1.3_{-0.08}^{+0.09} \mathrm{MeV}$.

Optical/NIR follow-up observations by GROND started 1.6 days post trigger. The afterglow was detected in all seven bands consistent with the reported redshift (Updike et al. 2009a). In addition, GROND imaged the field of GRB 090328 at 2.5, $3.5,4.5,6.5$ and 11.5 days after the trigger. The best optical position of the afterglow measured in the $r^{\prime}$ band against the USNO-B1 catalog is $\mathrm{RA}(\mathrm{J} 2000)=06: 02: 39.69, \operatorname{dec}(\mathrm{J} 2000)=$ $-41: 42: 54.9$ with systematic uncertainties of 0.3 in each coordinate. The position of the host galaxy is $\mathrm{RA}(\mathrm{J} 2000)=$ 06:02:39.69, $\operatorname{dec}(\mathbf{J} 2000)=-41: 52: 55.1$ and therefore the distance of the afterglow from the host is 0.14 . A finding chart of the afterglow is shown in Fig. 2.

Spectroscopy of the afterglow was performed on 2009 March 30.01 UT ( $\sim 1.6$ days post burst) using the FOcal Reducer and low dispersion Spectrograph 1 (FORS1; Appenzeller et al. 1998) at the $8 \mathrm{~m}$ ESO-VLT UT2 telescope (Programme ID: 083.D-0903). Observations were obtained using the $600 \mathrm{~B}$ and $600 R$ grisms, providing a spectral resolution of roughly $5 \AA$ full width at half maximum. Two $1800 \mathrm{~s}$ integrations were taken in each of the two grisms.

\subsection{GRB 090510}

At 00:23:00 UT on 10 May 2009 the Swift BAT triggered and located GRB 090510 (Hoversten et al. 2009). The burst also triggered Fermi/GBM (Guiriec et al. 2009) and other instruments (Golenetskii et al. 2009; Ohmori et al. 2009; Longo et al. 2009; Giuliani et al. 2010). The duration $\left(T_{90} \sim 2.1 \mathrm{~s}\right)$ and the negligible spectral lags below $\sim 1 \mathrm{MeV}$ of the event make 
it consistent with the class of short bursts (Abdo et al. 2009a). Significant emission was detected by the LAT (Ohno \& Pelassa 2009; Omodei et al. 2009). The time integrated spectrum using Fermi GBM and LAT data is best fit by two spectral components, a Band function with the Epeak $=3.9 \pm 0.3 \mathrm{MeV}$ and a powerlaw at high energies. The total isotropic energy is $(1.08 \pm 0.06) \times$ $10^{53}$ erg (10 keV-30 GeV) (Abdo et al. 2009a). The event was also detected at $5 \sigma$ significance at energies above $100 \mathrm{MeV}$ by AGILE GRID (Longo et al. 2009; Giuliani et al. 2010).

Swift slewed after $91 \mathrm{~s}$ and observations with the XRT and UVOT revealed an uncatalogued source (Marshall \& Hoversten 2009). The XRT light curve was reported to be fading (Grupe \& Hoversten 2009) and the UVOT afterglow candidate was confirmed by Olofsson et al. (2009) using the Nordic Optical Telescope. Kuin et al. (2009) report that the afterglow of GRB 090510 is detected in almost all UVOT filters which implies that the redshift is less than about 1.5 (Kuin \& Hoversten 2009). See also De Pasquale et al. (2010).

GROND imaged the field of GRB 090510 starting $6.18 \mathrm{~h}$ after the Swift and Fermi triggers. At that point the position of the GRB was becoming visible above the pointing constraints of the telescope (Olivares et al. 2009b). Therefore GROND images taken in the first two hours suffer from high airmass and moderate image quality. Observations continued for $4 \mathrm{~h}$ until local twilight and were resumed in the following night for $1.5 \mathrm{~h}$. In the first epoch, the afterglow is detected in the $g^{\prime} r^{\prime} i^{\prime} z^{\prime}$ optical channels, while the $J H K_{\mathrm{S}}$ band yield only upper limits and the second epoch only yield upper limits on the afterglow flux. The position of the optical transient associated with GRB 090510 is measured against USNO-B1 to $\mathrm{RA}(\mathrm{J} 2000)=22: 14: 12.54$, $\operatorname{dec}(\mathrm{J} 2000)=-26: 34: 59.1$ with errors of 0.5 in each coordinate. In both epochs a nearby, extended object with coordinates of $\mathrm{RA}(\mathrm{J} 2000)=22: 14: 12.56, \operatorname{dec}(\mathrm{J} 2000)=-26: 34: 58.7$ at a distance of 1 .'2 with respect to the afterglow is clearly detected. Given the low spatial separation both objects are blended in the first epoch. A finding chart of the field of GRB 090510 is shown in Fig. 3.

Spectroscopy of the host galaxy of GRB 090510 was performed ( 2.3 days post burst) using FORS2 (Appenzeller et al. 1998) at the $8 \mathrm{~m}$ ESO-VLT UT1 telescope (Programme ID: 083.D-0903(A)). Observations were obtained using the $300 I$ grism, providing a spectral resolution of $\approx 7 \AA F W H M$. Three $1800 \mathrm{~s}$ integrations were taken. The preliminary analysis and redshift $z=0.903$ was reported by Rau et al. (2009b). We note that the redshift of this galaxy is consistent with the afterglow colours detected by UVOT (Kuin et al. 2009). Additionally, spectroscopy of the source located $5^{\prime \prime}$ south of the GRB host galaxy was performed $\sim 1.3$ days post burst using FORS2 (300V grism), which yields a redshift consistent with that of the GRB host. Two $1800 \mathrm{~s}$ integrations were taken of this object.

\subsection{GRB $090902 B$}

At 11:05:08.31 UT on 2 September 2009 Fermi GBM triggered on a long, bright, hard burst GRB 090902B (trigger 273582310/090902462) (Bissaldi \& Connaughton 2009). The burst was localized by Fermi/LAT to RA(J2000), dec(J2000) = $265.00,27.33$ with a statistical uncertainty of only 0.06 degrees (de Palma et al. 2009b) and Target of Opportunity observations were initiated with the near-field instruments on Swift $\sim 12.5 \mathrm{~h}$ post-trigger. A candidate X-ray afterglow within the LAT error circle was reported by Kennea \& Stratta (2009) and

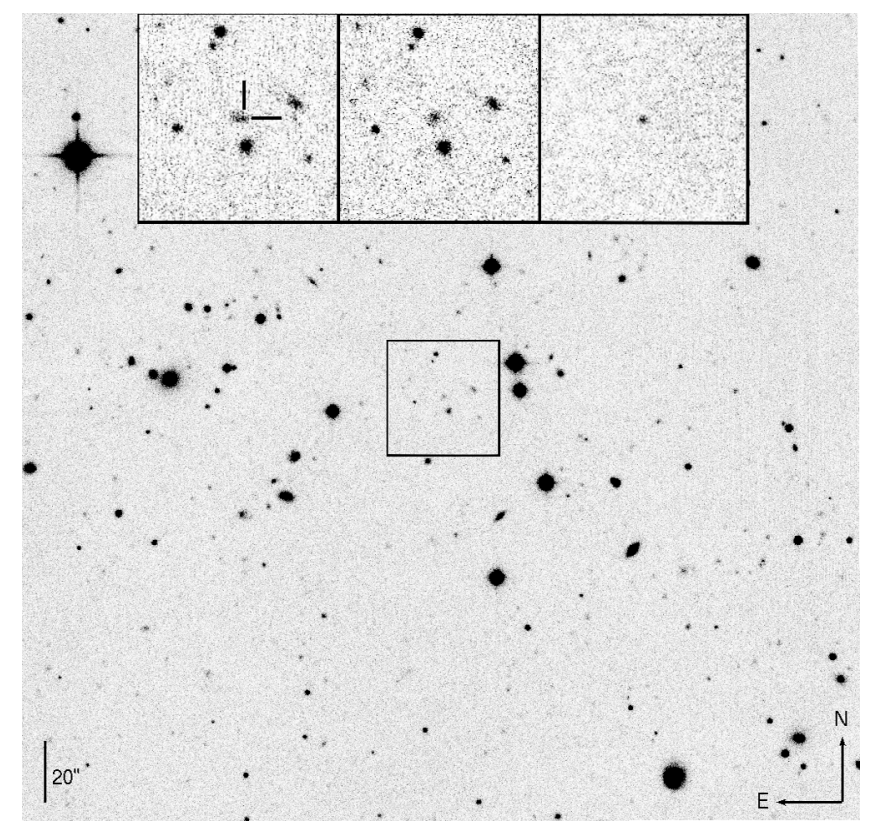

Fig. 3. GROND finding chart for the afterglow of GRB 090510. The main panel shows the field of GRB 090510 taken 1.4 days post burst. The field shown has $4^{\prime} \times 4^{\prime}$, where North is up and East is to the left. The inset in the top left shows a zoom into the afterglow region at $8.5 \mathrm{~h}$ after the burst, where the afterglow is indicated with two lines. The middle inset contains the same region with the host 1.4 days post burst. The top right inset is a difference image between the first and second epoch. All images are $i^{\prime}$ band.

subsequently confirmed to be fading (Stratta et al. 2009). Optical detections were reported by a number of observers (Swenson \& Siegel 2009; Swenson \& Stratta 2009; Perley et al. 2009a; Guidorzi et al. 2009; Pandey et al. 2009), in the NIR (Olivares et al. 2009a) and in the radio (van der Horst et al. 2009; Chandra $\&$ Frail 2009). The afterglow redshift of $z=1.822$ was obtained from an afterglow absorption spectrum by Cucchiara et al. (2009) using the GMOS spectrograph. Based on the spectral parameters (de Palma et al. 2009a), the isotropic energy released is $E_{\gamma, \text { iso }}=2.26 \times 10^{54}$ in the $1 \mathrm{keV}$ to $10 \mathrm{MeV}$ range and $3.54 \times 10^{54} \mathrm{erg}$ in the $1 \mathrm{keV}$ to $10 \mathrm{GeV}$ band. An excess of emission in addition to the Band function at both low energies $\$ 50 \mathrm{keV}$ and above $\gtrsim 100 \mathrm{MeV}$ was reported by Abdo et al. (2009b).

GROND imaged the field of GRB 090902B starting $\sim 13 \mathrm{~h}$ after the Fermi trigger when the position of the GRB was becoming visible above the pointing constraints of the telescope. At the time of the first epoch observations, only the LAT localization with 3.5 arcmin uncertainty was available. The afterglow location was outside the narrower $\left(5.4^{\prime} \times 5.4^{\prime}\right)$ optical field of view $(\mathrm{FoV})$ but within the larger NIR FoV $\left(10^{\prime} \times 10^{\prime}\right)$ (Olivares et al. 2009a). Observations continued in the following nights by GROND and an additional VLT/FORS2 $R$ band observation was executed at $\sim 23$ days post trigger (ESO DDT Proposal number: 283.D-5059). A finding chart of the field of GRB 090902B is shown in Fig. 4.

\section{Analysis}

GROND optical/NIR data were reduced and analyzed in standard manner using pyraf/IRAF tasks (Tody 1993). A detailed description of the reduction and relative photometry can be found in Krühler et al. (2008). In the afterglow-dominated regime we 


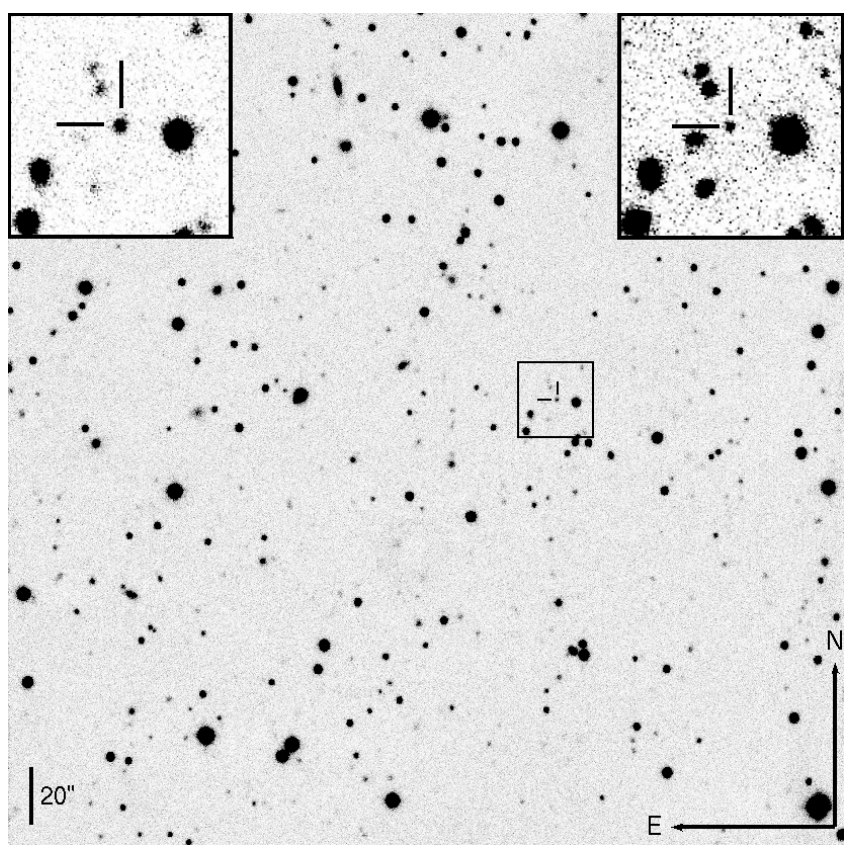

Fig. 4. GROND/VLT finding chart for the afterglow of GRB 090902B. The main panel shows the field of GRB 090902B in the $i^{\prime}$ band taken 1.5 days post burst. The shown field has $4^{\prime} \times 4^{\prime}$, where North is up and East is to the left. The afterglow position is indicated with two lines in each image. The inset in the top left shows a zoom into the afterglow region at the same epoch, where the afterglow was still bright. The top right inset shows a VLT $R$-band image taken 23 days after the trigger. Both insets were enhanced in contrast for demonstration purposes.

used PSF fitting techniques to derive the brightness of the optical transient. These were complemented by standard aperture photometry, which yields consistent results. The magnitudes of the GRB host galaxies were measured using aperture photometry with an appropriate aperture correction. In addition, SExtractor (Bertin \& Arnouts 1996) was used to perform host photometry, again with consistent results to the reported aperture photometry.

Absolute photometry in $g^{\prime} r^{\prime} i^{\prime} z^{\prime}$-bands was obtained against the $g^{\prime} r^{\prime} i^{\prime} z^{\prime}$ magnitudes of the Sloan Digital Sky Survey (SDSS DR7, Abazajian et al. 2009) for all bursts. SDSS field stars in the vicinity of GRB 090323 (Updike et al. 2009b) were used for GRBs 090323 and 090328. Both bursts were observed with GROND consecutively in 5 different nights, out of which 4 where photometric. The obtained calibration is consistent between all epochs. The field of GRB 090510 was calibrated against the primary Sloan Standards SA 114-750 and 114-650 and their SDSS field stars. Absolute photometry of the afterglow of GRB 090902B was obtained against a nearby SDSS field observed under photometric conditions. $J H K_{\mathrm{S}}$ absolute photometry was measured against the magnitudes of selected stars in the field of GRBs 090323, 090328, 090510 and 090902B from the 2MASS catalogue (Skrutskie et al. 2006). This procedure results in a typical absolute accuracy of $0.04 \mathrm{mag}$ in $g^{\prime} r^{\prime} i^{\prime} z^{\prime}$, $0.06 \mathrm{mag}$ in $J H$ and 0.08 in $K_{\mathrm{S}}$, which was added quadratically to the statistical error in the analysis of the broadband spectral energy distribution. TLS data of GRB 090323 was reduced in a standard manner with MIDAS and IRAF and calibrated against six SDSS stars in the field-of-view, where we derived $R_{\mathrm{C}}$ magnitudes using the transformations of Lupton from $2005^{1}$. The standard stars were measured with SExtractor, and

${ }^{1}$ http://www.sdss.org/dr7/algorithms/ sdssUBVRITransform.html\#Lupton2005

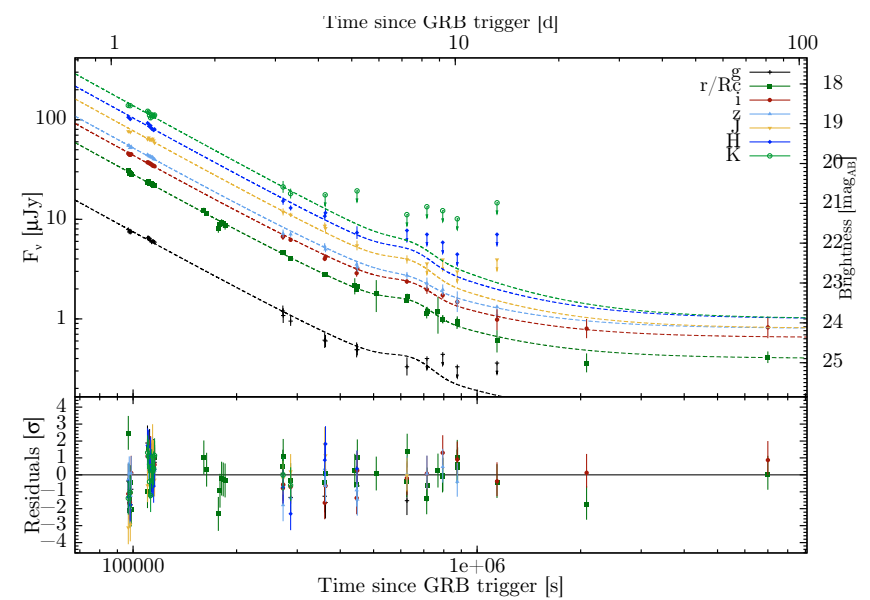

Fig. 5. GROND optical/NIR and TLS optical light curve of the afterglow of GRB 090323. The data are fitted with a power-law, superimposed bump and constant host component. Late upper limits are not shown to enhance clarity.

afterglow magnitudes were derived using aperture photometry. All magnitudes are corrected for the expected Galactic reddening in the direction of the bursts (Schlegel et al. 1998), which is $E_{B-V}=0.03 \mathrm{mag}$ for GRB 090323, $E_{B-V}=0.06 \mathrm{mag}$ for GRB 090328, $E_{B-V}=0.02 \mathrm{mag}$ for GRB 090510, and $E_{B-V}=0.04$ mag for GRB 090902B, respectively. All magnitudes are quoted in the $\mathrm{AB}$ system throughout this paper.

The FORS1+2 spectroscopy data were reduced with standard IRAF routines, and spectra were extracted using an optimal (variance-weighted) method. The observations were obtained with a long slit of 1.'0 width and corrected for slit losses due to finite slit width. Spectro-photometric calibrations were carried out using observations of the standard stars EG 274 and LTT 7379 (Hamuy et al. 1992) for the observations of GRB 090328 and GRB 090510, respectively.

\section{Results}

\subsection{The afterglow of GRB 090323}

\subsubsection{Light curve}

The GROND and TLS multicolor light curve of the optical/NIR afterglow starting at $T_{0}+95 \mathrm{ks}$ is presented in Fig. 5 and is well described with a single power-law plus a superimposed bump component and a constant host contribution dominating at later times $\left(\chi^{2}=161 / 138\right.$ d.o.f.). All available optical/NIR data were fitted simultaneously, allowing for a color change in the bump and host dominated regime. The initial power-law decay slope is $\alpha=1.90 \pm 0.01$, with no sign of an additional break at later times. The host magnitudes in the $g^{\prime} z^{\prime} J H K_{\mathrm{S}}$ bands are not constrained by the data and have been fixed. Similarly, the flare is unconstrained in the $H$ and $K_{\mathrm{S}}$ bands. The host galaxy of GRB 090323 was detected in the $r^{\prime}$ and $i^{\prime}$ bands but the stellar mass is not constrained by the optical identification (magnitudes $r^{\prime}=24.87 \pm 0.15$ and $i^{\prime}=24.25 \pm 0.18$ ) obtained by GROND because observations probe the rest-frame UV, where the massto-light ratio can vary by a factor of more than 100 .

\subsubsection{Spectral energy distribution}

The multi-band spectral energy distribution (SED) of the afterglow of GRB 090323 is shown in Fig. 6. It is well fit with a 


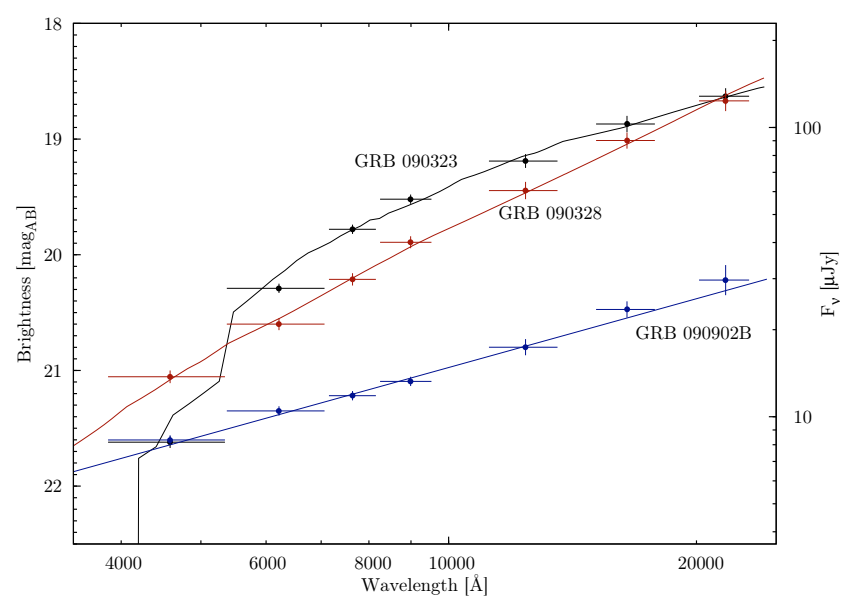

Fig. 6. GROND broadband spectral energy distribution of the afterglows of GRB 090323, GRB 090328 and GRB 090902B. Note that the $g^{\prime}$ band points for GRB 090323 and GRB 090902B are overlapping.

power-law of index $\beta_{\text {opt }}=0.65 \pm 0.13$ and a small amount of Small Magellanic Cloud (SMC)-like dust extinction (Bouchet et al. 1985) with $A_{V}^{\text {host }}=0.14_{-0.03}^{+0.04}\left(\chi^{2}=3.3 / 4\right.$ d.o.f. $)$. A pure power-law does not provide an acceptable fit $\left(\chi^{2}=26 / 5\right.$ d.o.f. $)$, and Large Magellanic Cloud (LMC) (Fitzpatrick 1986) and Milky Way (MW) (Seaton 1979) dust models are strongly ruled out because of the absence of the $2200 \AA$ feature, which would be in the $z^{\prime}$ band at this redshift. The flux difference between the extrapolation of the SED defined from $i^{\prime}$ to the $K_{\mathrm{S}}$ and the $g^{\prime}$ and $r^{\prime}$ bands is nicely consistent with the spectroscopic redshift of $z=3.57$. The final photo- $z$ obtained with the calibrated GROND data is $3.44_{-0.16}^{+0.18}$.

In the standard fireball model (see e.g. Piran 2004; Mészáros 2006 , for reviews) the spectral index, $\beta$ of the afterglow is connected to the temporal index, $\alpha$, via the closure relations. These depend on the type of circumburst medium, location of the characteristic frequencies in the synchrotron spectrum, and the evolutionary stage of the afterglow (e.g. Rees \& Meszaros 1998; Sari et al. 1998; Chevalier \& Li 2000; Zhang \& Mészáros 2004; Panaitescu 2005; Panaitescu et al. 2006; Zhang et al. 2006; Racusin et al. 2009). However, afterglow light curves often display complex temporal behaviour with shallow decays, plateaus, rebrightenings, flares or smooth breaks such that limited sampling strongly affects the inferred light-curve slope (e.g. Lipkin et al. 2004; Nousek et al. 2006; Evans et al. 2009; Greiner et al. 2009b). Hence, here and later, we derive the spectral index from the optical to NIR SED, and use the temporal index from the light curve fitting and the closure relations to obtain constraints on the jet properties. In particular, for a constant spectral index and value of $p$, the expected light curve slopes significantly differ ( $\Delta \alpha$ is between 0.5 and 1.3 depending on the model) between the pre and post jet break evolution, which facilitates a reliable discrimination between these two regimes.

For GRB 090323, the obtained spectral index $\beta_{\text {opt }}=$ $0.65 \pm 0.13$ is compatible with the observational frequency $v_{\mathrm{opt}}$ being between the typical synchrotron frequency $v_{\mathrm{m}}$ and the cooling frequency $v_{\mathrm{c}}$ for an ISM (density $n=$ const.) or wind $\left(n \propto r^{-2}\right)$ environment.

The power-law decay index of the light curve, $\alpha$, is expected to be $\sim 1$ in the pre-jet break, ISM case and is not compatible with the observations. A value of $\alpha \sim 2.3$ is obtained in a post-jet break evolution with significant lateral spreading of the ejecta, (both ISM and wind Chevalier \& Li 2000), and $\alpha \sim 1.7$ (ISM)

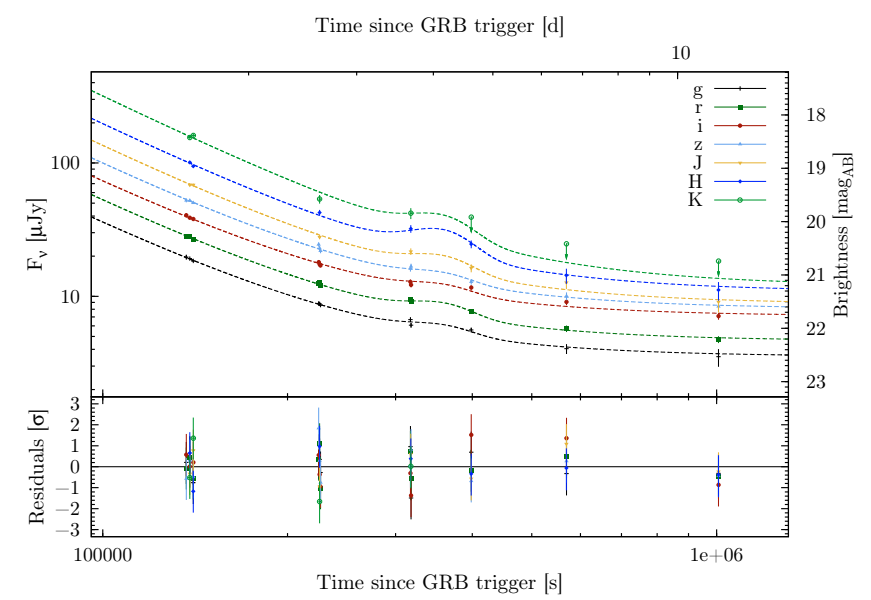

Fig. 7. GROND optical/NIR light curve of the afterglow of GRB 090328. The data are fitted with a power-law, superimposed bump and constant host component. All parameters are left free in the fit except the $K_{\mathrm{S}}$ band host magnitude, which is not constrained by the data.

or $\alpha \sim 2.0$ (wind) if lateral spreading of the ejecta is not considered (Panaitescu 2005). The latter case (post-jet break without spreading) is compatible within $1 \sigma$ with the observed $\alpha$ while the case with spreading is compatible at the $2 \sigma$ level. In the above cases the electron spectral index $p$ can be derived from $\beta=\frac{p-1}{2}$ and a value of $p=2.3 \pm 0.3$ is obtained.

The value of $\alpha$ for GRB 090323 indicates that the jet break occurred before the start of the observations. However it is not possible to distinguish between the ISM and wind environments. The post-jet evolution implies that the half opening angle of the jet is smaller than $\theta_{\text {jet }}^{\text {ISM }} \lesssim 2.1^{\circ}$ in an ISM-type circumburst environment (Sari et al. 1999; Frail et al. 2001). The beaming corrected energy emitted in $\gamma$-rays $E_{\gamma}(1 \mathrm{keV}$ to $10 \mathrm{GeV})$ of GRB 090323 is thus $E_{\gamma} \lesssim 3.3 \times 10^{51} \mathrm{erg}$. In a wind environment (Chevalier \& Li 2000) the opening angle of the jet would be $\theta_{\text {jet }}^{\text {wind }} \lesssim 1.1^{\circ}$ and the beaming corrected energy would be $E_{\gamma} \lesssim 1.0 \times 10^{51} \mathrm{erg}$. $(1 \mathrm{keV}$ to $10 \mathrm{GeV})$. The following values are assumed throughout the paper $A^{*}, n_{1}, \eta_{0} .2=1$.

\subsection{The afterglow and host galaxy of GRB 090328}

\subsubsection{Light curve}

The GROND multicolor light curve of the afterglow of GRB 090328 starting at $T_{0}+137 \mathrm{ks}$ is presented in Fig. 7 and is remarkably similar to that of GRB 090323 (Fig. 5). It is well described with a single power-law of index $\alpha=2.27 \pm 0.04$, which is typical of a post-jet break slope (Zeh et al. 2006), plus a superimposed bump component and a constant host contribution dominating at later times $\left(\chi^{2}=35 / 39\right.$ degrees of freedom). There is minor evidence for a slightly redder spectra in the bump component peaking at $\sim 350 \mathrm{ks}$ post burst, but given the uncertainties in the measurement however, the spectral index is consistent with the afterglow slope at the $2 \sigma$ level.

\subsubsection{Spectral energy distribution}

The broadband SED of the afterglow was constructed from data taken in the first epoch where the last epoch was used as a reference frame. Subtracting the host fluxes was performed using two methods: firstly the fluxes from the light curve fitting were used and directly subtracted from the first epoch magnitudes, 
where measurement errors were propagated accordingly. For the $K_{\mathrm{S}}$ band, where the host is undetected, we used the mean flux between the $H$ band detection and $K_{\mathrm{S}}$ band upper limits with errors over the complete range. The effect of the host magnitude error on the afterglow SED, however, is negligible as its uncertainty is dominated by the absolute photometric accuracy. Secondly, the reference frame was subtracted from the 1st epoch image in each band, and magnitudes were derived using the difference image directly. Both methods resulted in consistent results. As the second method tends to underestimate the true photon noise in the image, results from the direct flux difference with propagated errors are used in the following.

The afterglow SED was fitted using a power-law modified by dust reddening as shown in Fig. 6. A pure power-law provides a reasonably good fit to the data with a $\chi^{2}$ of $4.5 / 5$ d.o.f. The resulting power-law index of $\beta_{\text {opt }}=1.46_{-0.08}^{+0.07}$, however would be surprisingly red, both theoretically (Sari et al. 1998) and observationally (Kann et al. 2006, 2009a) if it were intrinsic to the afterglow. In fact, a small amount of extinction of $A_{V}^{\text {host }}=0.22_{-0.18}^{+0.06} \mathrm{mag}$ with a SMC type dust attenuation law provides a better fit $\left(\chi^{2}=2.6 / 4\right.$ d.o.f.) and a more typical spectral power-law index of $\beta_{\mathrm{opt}}^{\mathrm{SMC}}=1.19_{-0.19}^{+0.21}$. Though statistically not necessarily required, it seems thus very likely that there is mild reddening by dust in the circumburst environment. Due to the redshift of $z=0.7354$, the optical data obtained hardly probe the rest frame UV regime and do not probe the region of the $2200 \AA$ bump. Therefore, LMC and MW dust models cannot be distinguished and return comparable results, with spectral indices of $\beta_{\mathrm{opt}}^{\mathrm{LMC}}=1.07_{-0.12}^{+0.29}, \beta_{\mathrm{opt}}^{\mathrm{MW}}=1.16_{-0.21}^{+0.24}$ and $A_{V}^{\text {host }}$ between 0 and 0.4 mag.

A spectral index of $\beta_{\text {opt }} \sim 1.2$ is only consistent with temporal index $\alpha=2.27 \pm 0.04$ in a post jet break evolution and the ISM or wind, slow cooling case in the spectral regime above the cooling frequency $v_{\mathrm{opt}}>v_{\mathrm{c}}$ (Sari et al. 1998; Chevalier \& Li 2000; Zhang \& Mészáros 2004). In this case the electron energy index $p$ is $p=2 \beta=2.4_{-0.4}^{+0.4}$ as obtained from the spectral index. Hence, the observations indicate, that the jet break must have occurred before the first GROND observations. This yields an upper limit of $\theta_{\text {jet }}^{\mathrm{ISM}} \lesssim 5.5^{\circ}$ or $\theta_{\text {jet }}^{\text {wind }} \lesssim 4.2^{\circ}$ (Sari et al. 1999; Frail et al. 2001), which constrains $E_{\gamma}(1 \mathrm{keV}$ to $10 \mathrm{GeV})$ to $E_{\gamma} \lesssim 4.6 \times 10^{50} \mathrm{erg}$ for an ISM or $E_{\gamma} \lesssim 2.7 \times 10^{50} \mathrm{erg}$ for a wind type environment.

\subsubsection{The host galaxy of GRB 090328}

The optical spectrum of the afterglow of GRB 090328 is presented in Fig. 9 and shows a strong [OII] 33727 emission line, with a flux $f_{[\mathrm{OII}]}=(25.9 \pm 0.8) \times 10^{-17} \mathrm{erg} \mathrm{cm}^{-2} \mathrm{~s}^{-1}$. We possibly detect a weak, if there, [NeIII] $\lambda 3868$ emission with a flux nearly consistent with zero within the large uncertainties $f_{[\mathrm{NeIII}]}=(0.96 \pm 0.83) \times 10^{-17} \mathrm{erg} \mathrm{cm}^{-2} \mathrm{~s}^{-1}$.

The [OII] luminosity $L_{\text {[OII] }}=(6.47 \pm 0.21) \times 10^{41} \mathrm{erg} \mathrm{s}^{-1}$ would give a star formation rate $S F R=3.6 M_{\odot} \mathrm{yr}^{-1}$, not corrected for dust extinction (the SFR conversion derived for GRB hosts is from Savaglio et al. 2009). A mild dust extinction correction with $A_{V}^{\text {host }}=0.22$ gives $S F R=4.8 M_{\odot} \mathrm{yr}^{-1}$. This is about a factor of two higher than the mean $\langle S F R\rangle=$ $2.5 M_{\odot} \mathrm{yr}^{-1}$ derived for a sample 43 GRB hosts in the redshift interval $0<z<3.4$ (Savaglio et al. 2009), and about one order of magnitude higher than in the LMC. The spectrum as been corrected for slit losses, however we note that the star formation rate quoted above is a fraction of the total and that the true SFR may be slightly higher.
Table 1. Absorption lines in the afterglow spectrum of GRB 090328.

\begin{tabular}{|c|c|c|c|c|}
\hline Line & $\lambda_{\mathrm{obs}}$ & $z$ & $E W_{r}(\AA)$ & $\log N\left(\mathrm{~cm}^{-2}\right)$ \\
\hline FeII $\lambda 2344$ & 4067.0 & 0.7351 & $2.71 \pm 0.15$ & $16.07_{-0.36}^{+0.52}$ \\
\hline FeII $\lambda 2374$ & 4120.4 & 0.7356 & $2.20 \pm 0.15$ & \\
\hline FeII $\lambda 2381$ & 4133.9 & 0.7360 & $2.82 \pm 0.15$ & \\
\hline FeII $\lambda 2586$ & 4488.0 & 0.7355 & $2.69 \pm 0.18$ & \\
\hline FeII $\lambda 2600$ & 4511.2 & 0.7351 & $3.26 \pm 0.18$ & \\
\hline MnII $\lambda 2576$ & 4470.4 & 0.7354 & $1.16 \pm 0.16$ & $13.93 \pm 0.10$ \\
\hline MnII $\lambda 2594$ & 4500.9 & 0.7351 & $0.91 \pm 0.14$ & \\
\hline MnII $\lambda 2606$ & 4523.1 & 0.7356 & $0.79 \pm 0.15$ & \\
\hline MgII $\lambda 2796$ & 4851.7 & 0.7352 & $3.99 \pm 0.18$ & $>15.7$ \\
\hline MgII $\lambda 2803$ & 4864.3 & 0.7353 & $3.87 \pm 0.18$ & \\
\hline $\operatorname{MgI} \lambda 2852$ & 4949.6 & 0.7355 & $1.60 \pm 0.19$ & $13.38 \pm 0.12$ \\
\hline CaII $\lambda 3934$ & 5315.9 & 0.7353 & $>1.2$ & \\
\hline CaII $\lambda 3969$ & 5362.9 & 0.7353 & $1.68 \pm 0.19$ & $13.76 \pm 0.11$ \\
\hline
\end{tabular}

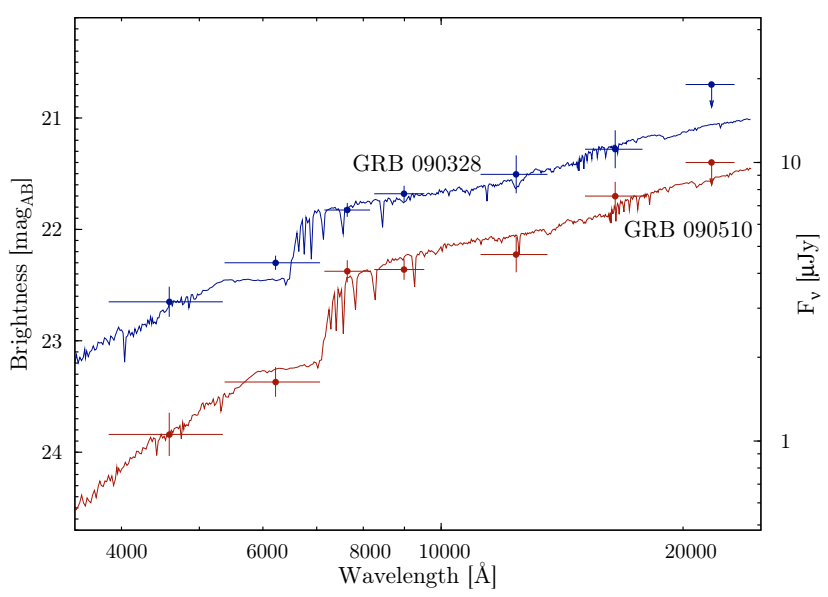

Fig. 8. Broadband spectral energy distributions of the host galaxies of GRB 090328 and GRB 090510 and host galaxy templates from hyperZ (Bolzonella et al. 2000).

The spectrum has a number of absorption lines at the same redshift of the [OII] line, associated with FeII, MnII, MgII, $\mathrm{MgI}$ and CaII. We derived column densities using the curve-ofgrowth analysis (Spitzer 1978), and cross-checked the results with the apparent optical-depth method (Savage \& Sembach 1991). Identification, rest-frame equivalent width $E W_{r}$ and measured column densities are in Table 1. The effective Doppler parameter given by the best fit is, independently for FeII and MnII, $b \simeq 78 \mathrm{~km} \mathrm{~s}^{-1}$. We used this value to derive the column density for MgI and CaII. We estimate a very uncertain ironto-manganese relative abundance $[\mathrm{Fe} / \mathrm{Mn}]=0.08_{-0.37}^{+0.53}$. No intervening absorption systems have been identified. The redshift obtained from the optical spectrum is $z=0.7354 \pm 0.0003$.

The SED of the host galaxy of GRB 090328 is presented in Fig. 8 and was fit using hyperZ (Bolzonella et al. 2000) and models from Bruzual \& Charlot (2003). The fitted galaxy spectral templates include elliptical, different types of early and late spirals, irregular and starburst galaxies at various ages and different star formation rates and solar metallicity. In addition, the spectral templates were modified by an extinction term, resembling the dust attenuation law of the SMC. The redshift of the galaxy was fixed at $z=0.7354$, which is consistent with the apparent $4000 \AA$ Balmer break being within the $r^{\prime}$ band.

The host galaxy of GRB 090328 is best fit with a starburst galaxy template, but all other templates provide acceptable fits. Similar to the afterglow SED, there is evidence for dust 


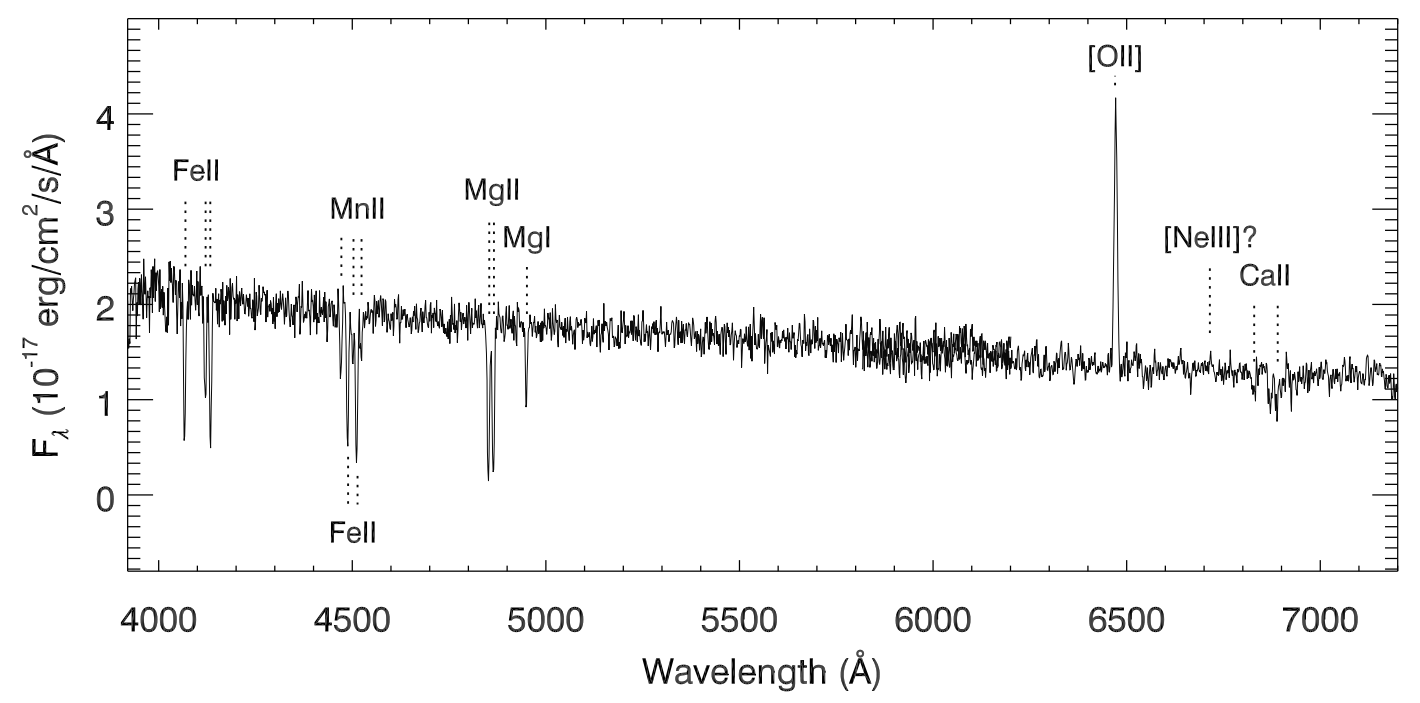

Fig. 9. VLT/FORS1 spectrum of the afterglow of GRB 090328 obtained $\sim 1.6$ days post-burst using the $600 B$ and $600 R$ grisms. Prominent absorption and emission lines are indicated and listed in Table 1.

reddening from the host observations of the order of $A_{V}^{\text {host }}=$ $0.5_{-0.3}^{+0.5}$ in the starburst galaxy (although $A_{V}^{\text {host }}$ is not well constrained), with a best-fit age of the dominant stellar population of $\sim 50$ Myr. By folding the restframe best-fit template spectrum with a typical $B$ and $K$ filter response, we derive absolute AB magnitudes of the host galaxy of GRB 090328 of $M_{B}=-20.67$ and $M_{K}=-20.17$, well within the samples of previous long GRB host galaxies (e.g., Christensen et al. 2004; Savaglio et al. 2009).

The stellar mass of the host can be approximated from the best fitting template to $M_{*} \simeq 10^{9.4} M_{\odot}$. When using the empirical relation derived by Savaglio et al. (2009) between $K$-band absolute magnitude and stellar mass:

$\log M_{*}=-0.467 \times M_{K}-0.179$,

we derive $M_{*} \simeq 10^{9.24} M_{\odot}$ with a typical uncertainty of a factor 2, consistent with the previous estimate and similar to the stellar mass of the LMC. Using the dust-corrected star formation rate $S F R=4.8 M_{\odot} \mathrm{yr}^{-1}$ (see note above on slit loss) and the above stellar mass, the specific star formation rate per unit mass rate is $2.8 \mathrm{Gyr}^{-1}$ again well within the sample of previous long burst host galaxies (Savaglio et al. 2009).

Using the galaxy distribution function in the corresponding redshift interval from $z=0.6-0.8$ from Willmer et al. (2006) to compare the host's absolute magnitudes to the population of field galaxies, the luminosity of the GRB host is estimated to $\sim 0.46-0.50 L_{*}$. We obtain a dust-corrected star formation rate per unit luminosity of $S F R / L_{B} \sim 10 M_{\odot} \mathrm{yr}^{-1} L_{*}^{-1}$ for this host galaxy. Hence, the specific star formation rate in the host galaxy of GRB 090328 is consistent with the values for the long bursts presented by Berger (2009).

\subsection{The afterglow and host galaxy of GRB 090510}

\subsubsection{Afterglow and host association}

The properties of the afterglow of GRB 090510 are not well constrained due its faintness at the time of the GROND observation and the blending with the nearby galaxy. Fitting the afterglow SED with a reddened power-law results in an unconstrained power-law index, and a $3 \sigma$ upper limit for the intrinsic extinction of $A_{V}^{\text {host }} \leq 1.1 \mathrm{mag}$, assuming an SMC-type extinction law. MW- or LMC-type extinction cannot be distinguished and result in comparable values for $\beta_{\text {opt }}$ and $A_{V}$.

The putative host galaxy at a distance of 1.'2, corresponding to a projected distance of $9.4 \mathrm{kpc}$ from the afterglow to the brightest emission in the nearby galaxy, has $r^{\prime}$ - and $z^{\prime}$-band magnitude of 23.4 and $22.3 \mathrm{mag}$, respectively and extends to the afterglow position in the GROND images of 0.9 seeing (Fig. 3). According to object counts from the Hubble Ultra Deep Field (Beckwith et al. 2006) and the GOODS (Giavalisco et al. 2004) one would statistically expect less than 0.008 unrelated objects of similar brightness at this distance to the afterglow, corresponding to a chance probability of $\lesssim 0.8 \%$. For comparison, the chance coincidence probability for the galaxies $\sim 5^{\prime \prime}$ south and $\sim 8^{\prime \prime}$ west of the afterglow are $\sim 7 \%$, and $\sim 30 \%$ respectively.

In the merger of two compact objects, the progenitors may be kicked outside their host galaxy at scales of 1-100 kpc, with a distribution peaking at several $\mathrm{kpc}$ for different galaxies and merger conditions (Fryer et al. 1999). Most of the convincing host galaxy associations however are found for host positions which coincide or overlap with the afterglow (e.g., Fox et al. 2005; Levan et al. 2006a; Kann et al. 2008; Troja et al. 2008). Short burst host samples show a typical offset of $0^{\prime \prime}-3^{\prime \prime}$ with clustering at small values (Berger et al. 2007; D'Avanzo et al. 2009) indicating that a large kick might not be a dominant process. Recently, based on HST observations of ten short burst hosts, Fong et al. (2010) report that the median offset of short GRBs from their host galaxies is $5 \mathrm{kpc}$ and based on a larger sample of short bursts they report that $25 \%$ of bursts have projected offsets of less than $10 \mathrm{kpc}$. The host galaxy of GRB 090510 certainly falls within this distribution. Berger et al. (2007) argue that even fainter, undetected galaxies at low redshifts $z \lesssim 0.5$ are very unlikely to host the dominant fraction of short GRBs. Also in the case of GRB 090510, direct observations of the optical afterglow place a strong constraint of $z<1.5$ of the redshift (Kuin et al. 2009). Given that the observed properties of the closeby galaxy fit very well into previous samples of short burst host galaxies in terms of brightness and redshift (e.g., Berger et al. 2007; Savaglio et al. 2009), and the probability of chance association is very low, this galaxy can be 


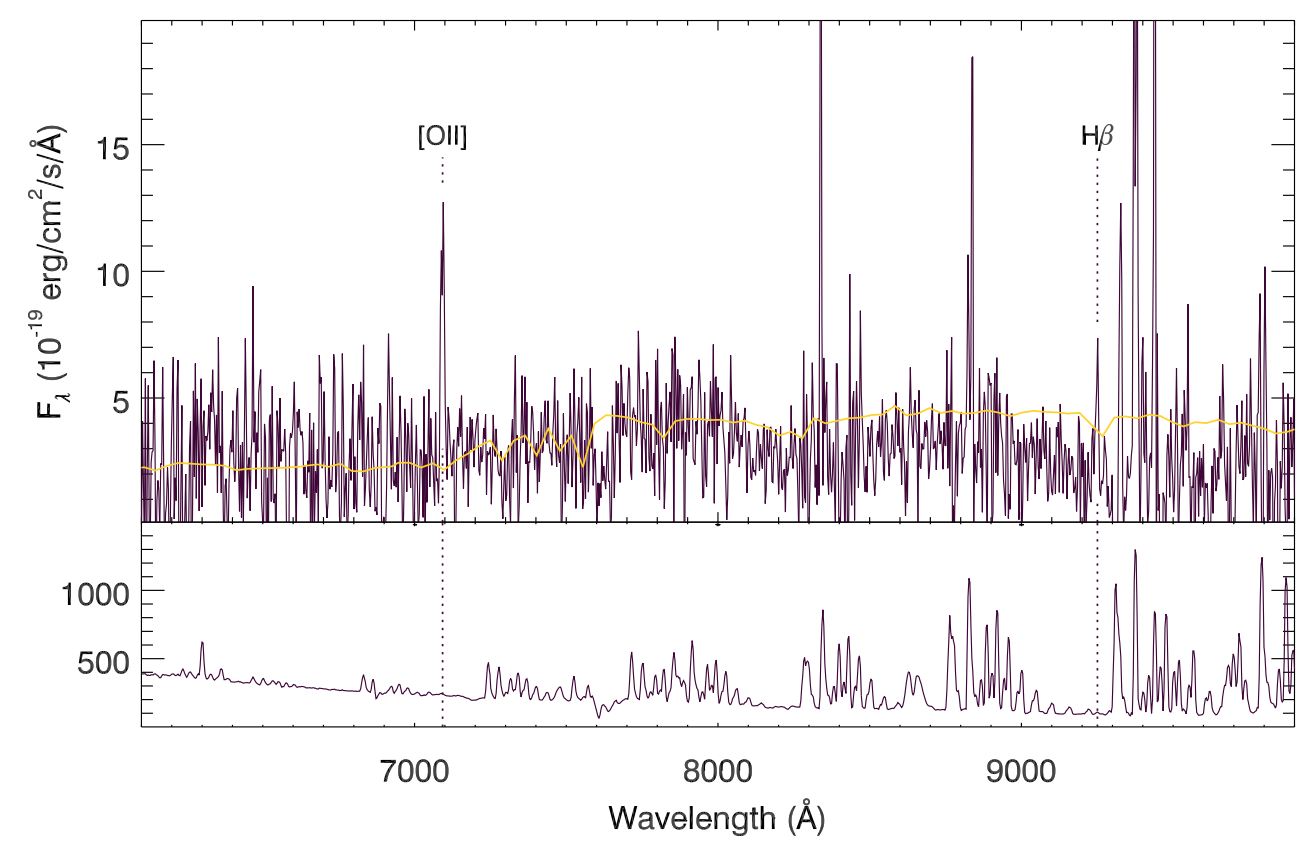

Fig. 10. VLT/FORS2 300I spectrum of the host galaxy of GRB 090510 obtained $\sim 2.3$ days post-burst (top panel) and corresponding noise spectrum (lower panel). The Sd galaxy template (yellow line; Polletta et al. 2007) in the top panel indicates the location of the $4000 \AA$ break. The [OII] and $\mathrm{H} \beta$ emission lines are marked and their fluxes listed in Table 2.

confidently associated with the burst and is hence very likely the host of GRB 090510.

We further report that the redshift of the galaxy $\sim 5^{\prime \prime}$ south obtained using VLT/FORS is the same as that of the host galaxy based on the detection of [OII] and $\mathrm{H} \beta$ emission lines. Using a distance scale of $7.8 \mathrm{kpc} /{ }^{\prime \prime}$ this translates to a projected distance of $\sim 40 \mathrm{kpc}$ and suggests that the host and this galaxy are part of a group or cluster association. Consequently in the unlikely event that this second galaxy were indeed the host galaxy, the distance to the source would therefore remain unchanged. The source to the east $\sim 8^{\prime \prime}$ away appears stellar and all the remaining nearby bright galaxies have chance coincidence probabilities of order $\sim 100 \%$.

\subsubsection{The host galaxy of GRB 090510}

The spectrum is presented in Fig. 10 and has a relatively weak [OII $] \lambda 3727$ emission line, with a flux $f_{[\mathrm{OII}]}=(1.30 \pm 0.15) \times$ $10^{-17} \mathrm{erg} \mathrm{cm}^{-2} \mathrm{~s}^{-1}$ (Table 2). The weaker $\mathrm{H} \beta$ flux is $f_{\mathrm{H} \beta}=$ $(0.50 \pm 0.15) \times 10^{-17} \mathrm{erg} \mathrm{cm}^{-2} \mathrm{~s}^{-1}$. The redshift obtained from the optical spectrum is $0.903 \pm 0.001$.

The $[\mathrm{OII}]$ and $\mathrm{H} \beta$ luminosities are $L_{[\mathrm{OII}]}=(5.4 \pm 0.6) \times$ $10^{40} \mathrm{erg} \mathrm{s}^{-1}$ and $L_{\mathrm{H} \beta}=(2.1 \pm 0.6) \times 10^{40} \mathrm{erg} \mathrm{s}^{-1}$, respectively. The star formation rate, not corrected for dust extinction, derived from the [OII] luminosity is $S F R=0.30 M_{\odot} \mathrm{yr}^{-1}$, consistent with the $S F R=0.26 M_{\odot} \mathrm{yr}^{-1}$ derived from the $\mathrm{H} \beta$ luminosity. The SFR conversions are those proposed by Savaglio et al. (2009) for GRB hosts. The derived value is one order of magnitude lower than the mean SFR measured in long GRB hosts (Savaglio et al. 2009) and consistent with relatively faint UV emission derived from the SED of the host (Fig. 8) and at the lower end of the distribution of SFRs of short GRBs (Berger 2009). Correcting for dust extinction yields a star formation rate of $\sim 0.60 M_{\odot} \mathrm{yr}^{-1}$. We note as in the case of GRB 090328, the spectrum as been corrected for slit losses, however we note that the star formation
Table 2. Emission lines detected in the spectrum of GRB 090510 corrected for Galactic extinction.

\begin{tabular}{lccc}
\hline \hline Line & $\lambda_{\mathrm{obs}}$ & $z$ & $\begin{array}{c}\text { Flux } \\
\left(10^{-17} \mathrm{erg} \mathrm{cm}^{-2} \mathrm{~s}^{-1}\right)\end{array}$ \\
\hline$[\mathrm{OII}] \lambda 3727$ & 7092.6 & 0.9030 & $1.30 \pm 0.15$ \\
$\mathrm{H} \beta$ & 9249.7 & 0.9028 & $0.50 \pm 0.15$ \\
{$[\mathrm{OIII}] \lambda 5007$} & n.d. & $\ldots$ & $<0.3$ \\
\hline
\end{tabular}

rate quoted above is a fraction of the total and that the true SFR may be slightly higher.

The SED fitting for the host of GRB 090510 has been performed in a similar manner to that of GRB 090328 (Sect. 4.2.3). The redshift of the galaxy was fixed to the spectroscopic value of $z=0.903$. Again all galaxy templates provide an acceptable fit, where the best fit is obtained with an elliptical galaxy. We note that the SFR is in the range of local star-forming elliptical galaxies (Huang \& Gu 2009). From the best fit we derive AB absolute magnitudes $M_{B}=-19.91$ and $M_{K}=-20.51$, and slight evidence for a dust extinction reddening similar to the SMC with a visual extinction of $A_{V}^{\text {host }}=0.7_{-0.4}^{+0.2}$. The stellar mass of the host can be estimated directly from the galaxy templates to $M_{*} \simeq 10^{9.6-10.2} M_{\odot}$. The dust-corrected star formation rate per unit mass is 0.04 to $0.15 \mathrm{Gyr}^{-1}$, significantly below that of the host galaxy of GRB 090328 .

Using the galaxy distribution function in the corresponding redshift interval from $z=0.8-1$ from Willmer et al. (2006), the luminosity of the GRB host is estimated to $\sim 0.26-0.30 L_{*}$, and we obtain a dust-corrected star formation rate per unit luminosity of $S F R / L_{B} \sim 2 M_{\odot} \mathrm{yr}^{-1} L_{*}^{-1}$ for this host galaxy, consistent with the albeit wide range of $S F R / L_{*}$ values for the short bursts presented by Berger (2009). 


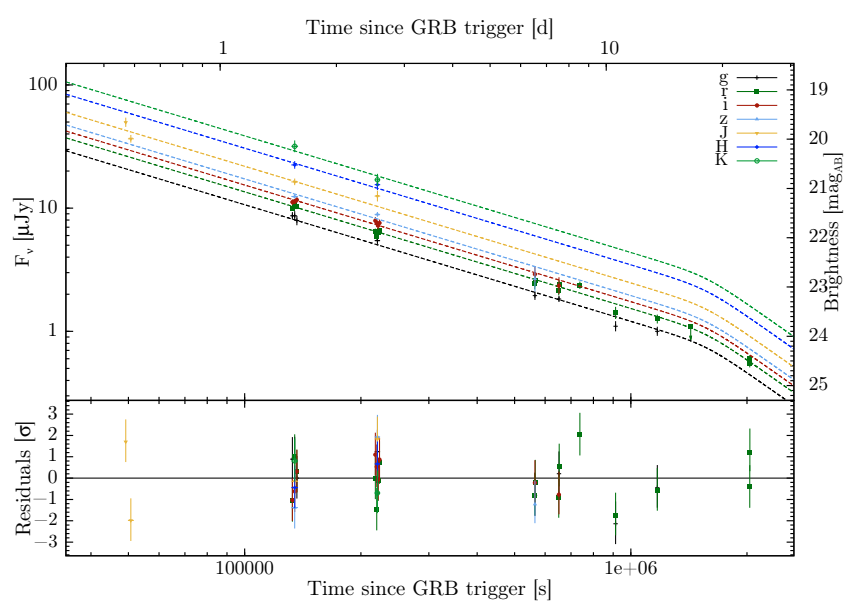

Fig. 11. GROND and VLT optical/NIR light curve of GRB 090902B. The data are fitted with a broken power-law. Due to the proximity of the source to the sun, the light curve coverage is sparse at later times, and the evolution is ambigous. There is however indication of a break at around $1.5 \mathrm{Ms}$ post burst. Shown is the fit with a fixed late slope and relatively sharp break.

\subsection{The afterglow of GRB 090902B}

\subsubsection{Light curve}

The early ( $\left.t<T_{0}+1.10 \mathrm{Ms}\right)$ GROND multi-color light curve of the optical/NIR afterglow of GRB 090902B is well fit by a single power-law of index $\alpha_{1}=0.94 \pm 0.02$. A late epoch of imaging obtained with the VLT 23 days after the burst indicates a break in the light curve at the $2 \sigma$ level. No further followup observations could be performed due to the proximity of the GRB field to the Sun. Consequently the light curve coverage at late times is sparse and the evolution not fully conclusive. Fixing the late slope to a conventional post jet break slope of $\alpha_{2}=2.2$ with a relatively sharp break as shown in Fig. 11 results in a break time of $t \sim 1.5 \mathrm{Ms}\left(\chi^{2}=43 / 35\right.$ d.o.f. $)$.

\subsubsection{Spectral energy distribution}

The optical/NIR SED of the afterglow of GRB 090902B is well described with a simple power-law template (Fig. 6). The spectral index is $\beta_{\text {opt }}=0.79_{-0.19}^{+0.05}$, with $3 \sigma$ upper limits of the intrinsic visual extinction of $A_{V}^{\text {host }} \lesssim 0.36$ with an SMC/LMC type reddening and $A_{V}^{\text {host }} \lesssim 0.24$ assuming a MW-like dust attenuation law. This is in agreement with the light curve slope of $\alpha \sim 0.94$ in the fireball model, which is typical for a pre-jet break evolution with an ISM surrounding and with an observational frequency between $v_{\mathrm{m}}$ and $v_{\mathrm{c}}$. Following Sari et al. (1999) and Frail et al. (2001), the half opening angle for a break time of $t \sim 1.5 \mathrm{Ms}$ would be $7.2^{\circ}$, or setting $t_{\text {break }} \gtrsim 1.1 \mathrm{Ms}$ as a conservative lower limit $\theta_{\text {jet }} \gtrsim 6.4^{\circ}$. Together with the energetics of the prompt emission, this implies a beaming corrected energy of $E_{\gamma} \gtrsim 2.2 \times$ $10^{52} \mathrm{erg}$. We note that a wind environment would significantly relax the lower bounds on the opening angle and thus $E_{\gamma}$, however it is not consistent with the expectations for $\alpha$ and $\beta_{\text {opt }}$ in the standard models. The power-law index $p$ of the electron population is then $p=2.58_{-0.38}^{+0.10}$ as obtained from the spectral index which is in reasonable agreement with the values estimated from the early LAT emission under the assumption that it is generated by an external forward shock (Kumar \& Barniol Duran 2009a).

\section{Discussion}

We have presented the optical and NIR follow-up observations of four bursts with high energy emission detected by Fermi/LAT and spectroscopic investigations of two of those events. Since the end of the commissioning phase Fermi/GBM has detected several hundred GRBs, many of which were in the field of view of the LAT instrument, however only fourteen of those have been also detected in by LAT (up to the end of January 2010). Follow-up of these bursts is of special interest due to the broadband coverage of the prompt emission ( $\mathrm{keV}$ to $\mathrm{GeV}$ ) which allows the spectral properties and energetics of these events to be constrained. It is noteworthy that the redshifts of bursts detected in the $\mathrm{GeV}$ regime by the LAT range from the relatively low-redshift GRB 090328 to the high-reshift of GRB 080916C and that the long bursts have high fluences and very high isotropic gamma-ray energies (although GRB 090328 has a smaller isotropic energy release due to its lower redshift). The properties of these events presented in this paper and another Fermi/LAT event, GRB 080916C, including their afterglows and energetics are summarized in Table 3.

Three of the bursts presented in this paper belong to the class of long gamma-ray bursts while GRB 090510 is a short burst. The existence of two classes of gamma-ray bursts has been established for some time (e.g., Mazets et al. 1981; Norris et al. 1984; Hurley 1992; Kouveliotou et al. 1993). It is generally accepted that long GRBs have their origins in massive star progenitors because of their association with core-collapse supernovae (SNe) (e.g. Galama et al. 1998; Hjorth et al. 2003; Malesani et al. 2004; Zeh et al. 2004) and occurrence in starforming galaxies (Bloom et al. 2002) and in highly star-forming regions therein (Fruchter et al. 2006). For a recent review of gamma-ray bursts see Vedrenne \& Atteia (2009).

The origin of short GRBs is still open, with mergers of compact objects being the leading concept (e.g., Eichler et al. 1989; Gehrels et al. 2005; Hjorth et al. 2005; Fox et al. 2005; Levan et al. 2006b). For a recent review of short bursts and their progenitors see Nakar (2007) and Lee \& Ramirez-Ruiz (2007). In contrast to long GRBs, the afterglows of the short bursts are generally fainter (Kann et al. 2008; Nysewander et al. 2009), making it difficult to obtain an absorption line spectrum of the afterglow itself. Almost exclusively, the distances to short bursts are obtained by associations with and observations of the (putative) host galaxies (e.g., Berger 2009; Graham et al. 2009). See however Levan et al. (2007). One possible exception is the case of GRB 090426 at $z=2.609$ (Levesque et al. 2010; Antonelli et al. 2009; Zhang et al. 2009) for which absorption line spectroscopy of the afterglow was successfully obtained, but may be considered on balance by the authors to be associated with the population of long gamma-ray bursts. The host galaxies of the short bursts were found initially to be mostly elliptical galaxies, e.g., GRB 050509B (Gehrels et al. 2005; Bloom et al. 2006) and GRB 050724 (Berger et al. 2005; Gorosabel et al. 2006) and that an old progenitor population was required. However, as the sample has since increased, all types of galaxies, from elliptical to star-forming, have been found to be associated with short bursts and the majority of short bursts are now found to reside in star forming galaxies (e.g., Berger et al. 2007; Berger 2009; Prochaska et al. 2006). A comparison of the short and long burst hosts by Berger (2009) revealed that the former have higher luminosities, and that it is unlikely that both samples are drawn from the same underlying galaxy distribution $\left(p=10^{-3}\right)$ and that the short burst hosts properties match well to field galaxies in the range $z \sim 0.1-1$. 
S. McBreen et al.: Optical and NIR follow-up of Fermi/LAT GRBs

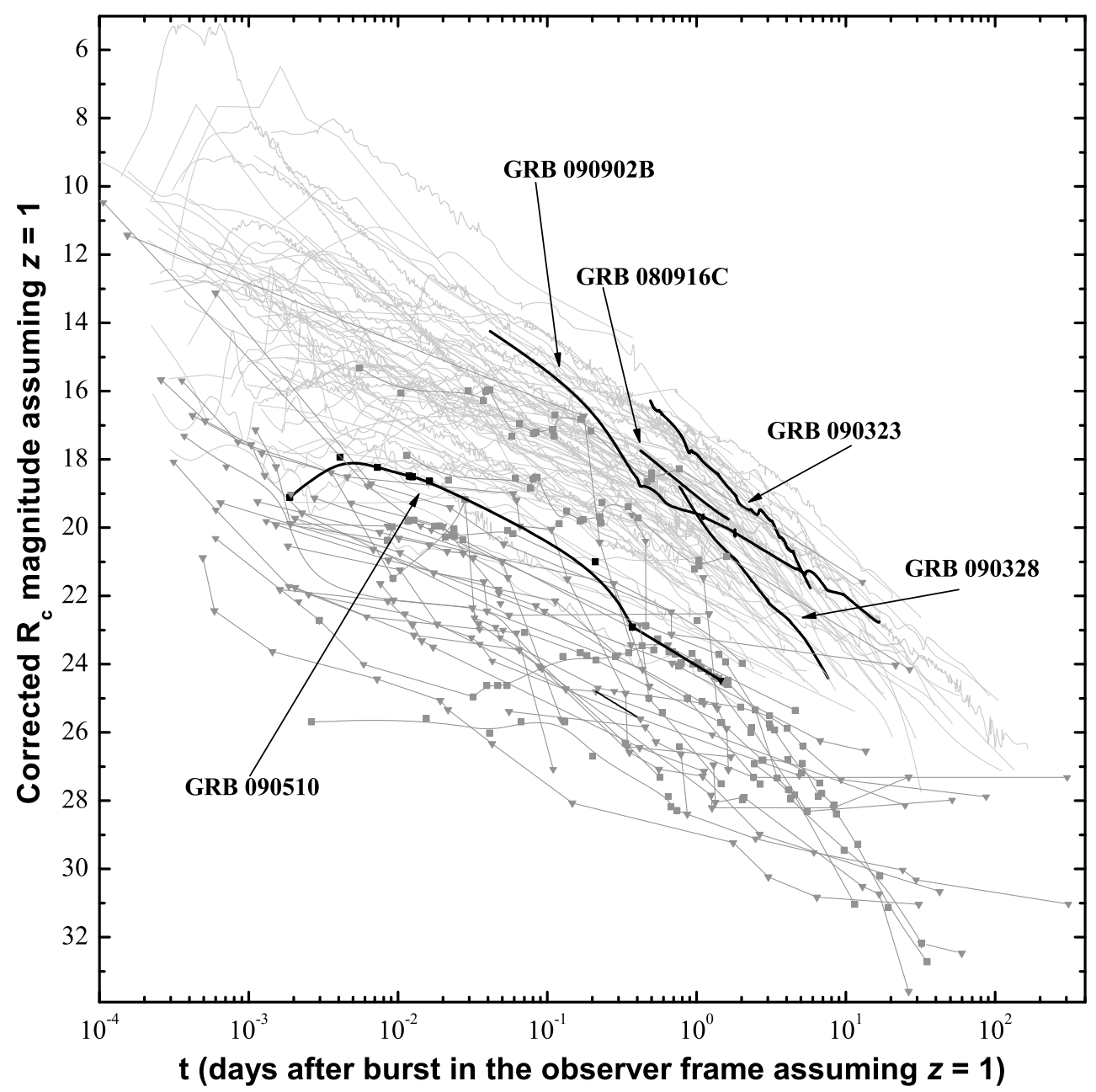

Fig. 12. The afterglows of the Fermi/LAT-detected GRBs in comparison with a sample of over 120 well observed afterglows detected until May 2009 (Kann et al. 2006, 2009a, 2008). GROND and TLS data only are presented for the the long Fermi bursts while the early data points from GRB 090510 are from UVOT (Kuin \& Hoversten 2009) and include a point from Olofsson et al. (2009). All afterglows have been corrected for Galactic extinction and for host-galaxy contribution, where applicable. All afterglows have been additionally corrected for host-galaxy extinction, and have been shifted to a redshift of $z=1$. Long GRBs are shown as light grey lines, whereas short GRBs are thicker grey lines with symbols. Squares are detections, and downward pointing triangles upper limits.

Table 3. Summary of the burst properties of five Fermi/LAT bursts.

\begin{tabular}{|c|c|c|c|c|c|c|c|c|c|}
\hline Burst & Redshift & $\begin{array}{c}E_{\gamma, \text { iso }}{ }^{\prime} \\
\text { erg }\end{array}$ & $\alpha$ & $\beta_{\mathrm{opt}}$ & Template & $A_{V}^{\text {host }}$ & $\theta_{\substack{\text { jet } \\
\circ}}^{\mathrm{ISM}}$ & $\begin{array}{l}E_{\gamma}{ }^{a} \\
\text { (erg) }\end{array}$ & $\begin{array}{c}\mathrm{SFR}^{b} \\
\left(M_{\odot} \mathrm{yr}^{-1}\right)\end{array}$ \\
\hline GRB $090323^{c}$ & $3.57^{1}$ & $5.1 \times 10^{54}$ & $1.90 \pm 0.01$ & $0.65 \pm 0.13$ & SMC & $0.14_{-0.03}^{+0.04}$ & $\lesssim 2.1^{\circ}$ & $\$ 3.3 \times 10^{51}$ & $\cdots$ \\
\hline GRB 090328 & 0.7354 & $1.0 \times 10^{53}$ & $2.27 \pm 0.04$ & $1.19_{-0.21}^{+0.24}$ & SMC & $0.22_{-0.18}^{+0.06}$ & $\lesssim 5.5^{\circ}$ & $\lesssim 4.6 \times 10^{50}$ & 4.8 \\
\hline GRB 090902B & $1.822^{2}$ & $3.5 \times 10^{54}$ & $0.94 \pm 0.02$ & $0.79_{-0.19}^{+0.05}$ & SMC/LMC & $\$ 0.34$ & $\gtrsim 6.4^{\circ}$ & $\gtrsim 2.2 \times 10^{52}$ & $\cdots$ \\
\hline GRB $080916 C^{3}, c$ & $4.35^{3}$ & $8.8 \times 10^{54}$ & $1.40 \pm 0.05$ & $0.38_{-0.19}^{+0.20}$ & $\cdots$ & $\cdots$ & $\gtrsim 6^{\circ}$ & $\gtrsim 4.8 \times 10^{52}$ & $\cdots$ \\
\hline GRB 090510 & 0.903 & $1.1 \times 10^{53}$ & $\ldots$ & $\ldots$ & $\cdots$ & $\leq 1.1$ & $\cdots$ & $\ldots$ & 0.30 \\
\hline
\end{tabular}

References. (1) Chornock et al. (2009); (2) Cucchiara et al. (2009); (3) Greiner et al. (2009a).

Notes. ${ }^{(a)} E_{\gamma, \text { iso }}$ values are quoted in the energy range $1 \mathrm{keV}-10 \mathrm{GeV}$, with the exception of GRB 080916C (10 keV-10 GeV: Abdo et al. 2009a) and GRB $090510\left(10 \mathrm{keV}-30 \mathrm{GeV}\right.$ : Abdo et al. 2009b). ${ }^{(b)}$ The true SFR may be larger than measured because of slit losses. ${ }^{(c)}$ The opening angle and $E_{\gamma}$ are lower in a wind medium for GRB 090323 and $080916 \mathrm{C}$.

\subsection{Afterglow properties and energetics}

The bursts were followed up in the optical and NIR starting at times ranging from $6 \mathrm{~h}$ to 1.6 days post trigger. In all cases a bright afterglow was detected and followed for many days, with the exception of GRB 090510 which was only detected on the first night post-trigger. The afterglow light curves of the three long bursts are well sampled in the seven GROND bands. The two March events are well fit by power-laws with indices consistent with post-jet break models constraining the opening angle to be before the start of GROND observations. In the case of GRB 090323 the opening angle $\left(\theta_{\text {jet }}^{\mathrm{ISM}}\right)$ is less than $2.1^{\circ}$ while for 
GRB 090328 the observations began later and the opening angle $\left(\theta_{\text {jet }}^{\mathrm{ISM}}\right)$ is less than $5.5^{\circ}$. The slope of the afterglow decay of GRB 090902B is flatter and consistent with a pre-jet break value, constraining the opening angle $\left(\theta_{\text {jet }}^{\mathrm{ISM}}\right)$ of the jet to be $\gtrsim 6.4^{\circ}$.

Knowledge of the opening angle allows us to correct the isotropic energy and calculate limits on $E_{\gamma}$, the beaming corrected energy release in $\gamma$-rays (Sari et al. 1999; Frail et al. 2001). The $E_{\gamma}$ of GRBs 090323 and 090328 is in a range compatible with the majority of GRBs observed to date. In the case of GRB 090902B, the late break places at least a lower limit on the opening angle and therefore a very large beaming corrected energy output of $\gtrsim 2.2 \times 10^{52} \mathrm{erg}$ is obtained. To date this is one of the largest values of $E_{\gamma}$ measured and indicates that the large $E_{\gamma, \text { iso }}$ values of Fermi/LAT GRBs are not always due to a narrow opening angle where we happen to be located in the cone. The value of $E_{\gamma}$ obtained for the three long bursts ranges from less than $5 \times 10^{50} \mathrm{erg}$ to greater than $2 \times 10^{52} \mathrm{erg}$, spanning over a factor of at least 30 . Values in excess of $10^{52}$ erg have been reported for another Fermi/LAT detected burst (Greiner et al. 2009a) and a number of Swift bursts (Cenko et al. 2010a) and indicate that the distribution of $E_{\gamma}$ is broad and not compatible with a standard candle (Frail et al. 2001; Bloom et al. 2003). Furthermore these very luminous GRBs with high values of the redshift corrected $E_{\text {peak }}$ are not compatible with the $E_{\text {peak,i }}-E_{\gamma}$ relationship as shown in Fig. 13 which was obtained for GRBs with in general lower values of the peak energy (Ghirlanda et al. 2004; Campana et al. 2007). While being consistent with the Amati $\left(E_{\text {peak,i }}-E_{\text {iso }}\right)$ relation at the $2 \sigma$ level (see also Amati et al. 2009), albeit with a large scatter (see also e.g. Butler et al. 2007), all four LAT bursts are inconsistent with the Ghirlanda relation (Ghirlanda et al. 2004) at $\gtrsim 2 \sigma$ using a standard approach. Two GRBs (080916C and 090902B) have too large beaming corrected $E_{\gamma}$ for their $E_{\text {peak,i, }}$, while $E_{\text {peak,i }}$ for GRBs 090323 and 090328 is too high to fit the Ghirlanda relation. In the earlier case, subtracting the energy in the additional power-law component of the prompt emission (Abdo et al. 2009b), a wind environment, or a two-component model could possibly relax the constraints on $E_{\gamma}$ directly (extracomponent) or indirectly by reducing the lower limit on the opening angle (Wind-case), or by disconnecting the beaming of the prompt emission from the late afterglow (two-component model). Although somewhat contrived, multi-component jets (e.g. Berger et al. 2003; Racusin et al. 2008; Krühler et al. 2009a) would allow the $\gamma$-ray emission to be tighter collimated than the late afterglow, and hence reduce the measure on the opening angle obtained from the afterglow light-curve to an upper limit. Similar arguments can not be invoked for GRBs 090323 and 090328, where the latter one in particular is a significant outlier from the $E_{\text {peak,i }}-E_{\gamma}$ relationship (Fig. 13).

\subsection{Host properties}

Host galaxies were detected for two of the long bursts, GRBs 090323 and 090328, and the short burst GRB 090510. The host galaxy of GRB 090323 was detected in the $r^{\prime}$ and $i^{\prime}$ bands but further constraints could not be obtained due to the faintness of the source and the high redshift. The host galaxy of GRB 090328 is best fit with a burst galaxy template (although all other templates provide acceptable fits) and the absolute magnitudes are comparable with previous long GRB host galaxies (e.g., Christensen et al. 2004; Savaglio et al. 2009). A dust-corrected star-formation rate of $4.8 M_{\odot} \mathrm{yr}^{-1}$, as well as the star formation rate per unit mass $\left(2.8 \mathrm{Gyr}^{-1}\right)$ and per unit

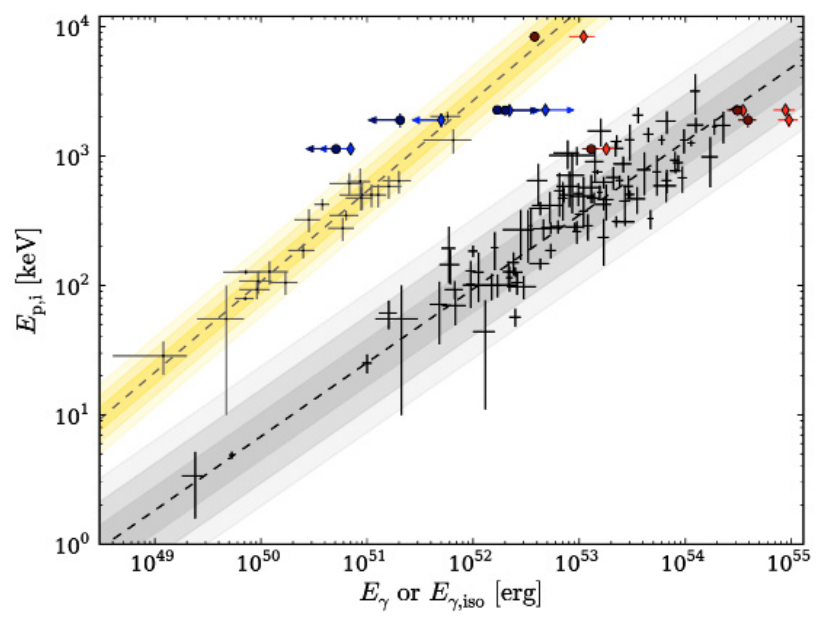

Fig. 13. Amati and Ghirlanda relation challenged by Fermi/LAT bursts. The black crosses, the black dashed line, and the grey shaded areas represent the data and best-fit $E_{\mathrm{peak}, \mathrm{i}}-E_{\mathrm{iso}}$ relation and its $1 \sigma, 2 \sigma$ and $3 \sigma$ scatter as compiled in Amati et al. 2008. The grey crosses, the grey dashed line, and the golden shaded areas represent the data and bestfit $E_{\text {peak,i }}-E_{\gamma}$ relation and its $1 \sigma, 2 \sigma$ and $3 \sigma$ scatter as compiled in Ghirlanda et al. 2007. Red points are the 5 Fermi/LAT bursts with respect to the Amati relation. The red circles are calculated in the bolometric energy range ( $1 \mathrm{keV}$ to $10 \mathrm{MeV}$ ) and the red diamonds are calculated over the energy ranges quoted in Table 3 . The four long LAT bursts are consistent with it at the $\$ 2 \sigma$ level. Blue points with arrows are the limits on the four long Fermi/LAT bursts with respect to the Ghirlanda relation. The blue circles symbols indicate the data in the bolometric energy range ( $1 \mathrm{keV}$ to $10 \mathrm{MeV})$ and the diamonds are again the values from Table 3. Over the bolometric energy range all four are inconsistent with the relation at $\gtrsim 2 \sigma$ level and over the wider energy range, three are inconsistent with it at the $\gtrsim 3 \sigma$ level and GRB 090323 is consistent.

luminosity $\left(\sim 10 M_{\odot} \mathrm{yr}^{-1} L_{*}^{-1}\right)$ derived from the afterglow spectrum are consistent with the long burst sample (Savaglio et al. 2009; Berger 2009). The host galaxy of GRB 090510 was detected by GROND and a spectrum was obtained by the VLT. A star formation rate of $0.30 M_{\odot} \mathrm{Gyr}^{-1}$ (not corrected for dust) was derived and the host galaxy is best fit by an elliptical template, however all templates provide acceptable fits. The dust-corrected star formation rate per unit luminosity of $\sim 2 M_{\odot} \mathrm{yr}^{-1} L_{*}^{-1}$ for this host galaxy is consistent with the values for the short bursts presented by Berger (2009). The properties of the host galaxies of two bursts with high-energy emission detected by Fermi/LAT, the long burst GRB 090328 and the short burst GRB 090510, are not exceptional with respect to pre-Fermi samples.

\subsection{Optical afterglows of LAT-detected GRBs in comparison with the previous sample}

With knowledge of the distance from the redshift determination and the local dust extinction from the SED fitting, we are able to compare the afterglows of our sample of LAT-detected GRBs (where we also include GRB 080916C, Greiner et al. 2009a) with a large sample of well observed GRB afterglows presented in Kann et al. (2006) (pre-Swift long GRBs), Kann et al. (2009a) (Swift-era long GRBs) and Kann et al. (2008) (Swift-era short GRBs). Using the method detailed in Kann et al. (2006), the afterglows are corrected for host-galaxy extinction and all shifted to a common redshift of $z=1$, so they can be compared directly both in terms of temporal evolution as well as luminosity. The 


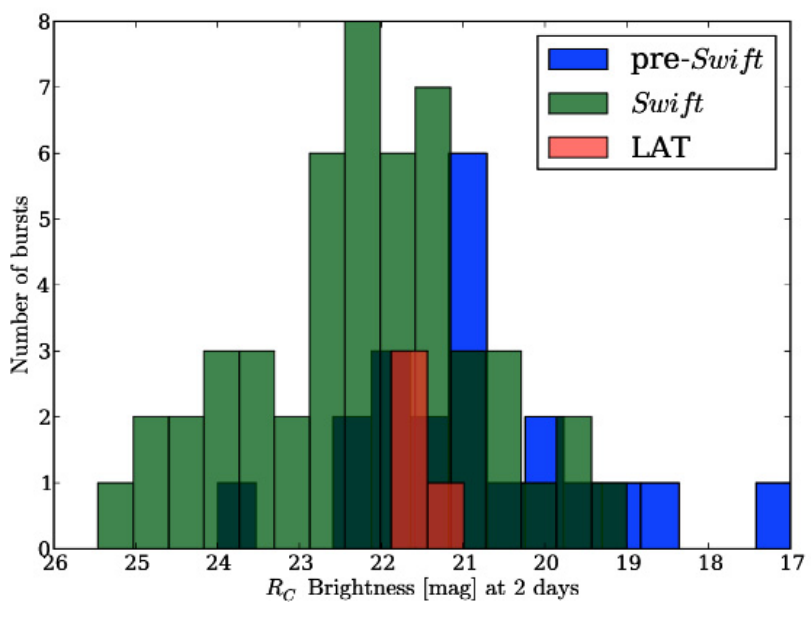

Fig. 14. The afterglow brightness at day 2 of four long Fermi/LATdetected GRBs in comparison with a sample of over 70 well observed afterglows of long GRBs detected until May 2009 (Kann et al. 2006, 2009a). The comparison sample comprises 50 Swift-era and 21 preSwift afterglows.

LAT-detected-GRB afterglows are shown in comparison to the total sample in Fig. 12.

In comparison to the sample of long GRB afterglows, the three afterglows presented in this work as well as GRB 080916C are seen to be diverse. The optical afterglow of GRB 090323, especially at early times, is one of the most luminous afterglows ever detected. The steep decay from discovery on gives it a more typical luminosity at later times. The afterglow of GRB 080916C lies close to the mean of the sample distribution. At discovery, the afterglow of GRB 090328 has a similar luminosity, but again, a steep decay from the onset implies that it becomes much less luminous at later times. The afterglow of GRB 090902B has a flatter slope and the very late break causes it to be one of the most luminous afterglows known at late times.

Observationally, however, the four optical afterglows of the long Fermi/LAT bursts are exceptionally bright. The histogram of observed magnitudes from the sample in Fig. 12 is shown in Fig. 14 at a common time of 2 days after the trigger in the observers frame. At this time, all four bursts are brighter than the mean brightness $\left(R_{\mathrm{C}}=22.2\right)$ of the Swift-era comparison sample. Furthermore, the sample is not complete, comprising 50 out of a total of 370 bursts with X-ray afterglows as a rough indicator for observability between the launch of Swift and May 2009. Accounting for the observational bias, and assuming in the extreme case that the comparison sample is complete at the bright end of the distribution, then all four LAT afterglows would lie in the brightest $5 \%$, with GRB 090328 being the brightest (top 2\%, i.e. 8th brightest of Swift-era or 16th of all afterglows).

In comparison to the afterglows of other short GRBs (grey lines), it can be seen that the afterglow of GRB 090510 is among the most luminous (in agreement with its extreme prompt energy release, Abdo et al. 2009a, and the correlation between prompt energy release and afterglow luminosity, Kann et al. 2008; Nysewander et al. 2009; Gehrels et al. 2008), at least at early times. After just a few hours, it goes over into a steep, probably post-jet break decay, and at one day, its luminosity is comparable to the few other short GRB afterglows detected at this time. The upper limit derived from the second GROND epoch is not restrictive.

The host galaxy extinction found in all three long GRB afterglows lies within the range of $A_{V} \approx 0.2$ which is typical for bright optical/NIR afterglows (Kann et al. 2009a), with no extinction detected in the case of GRB 080916C (Greiner et al. 2009a).

The intrinsic spectral slopes $\beta_{\text {opt }}$ are also diverse, with that of GRB 090328 being a typical value for an afterglow with a cooling break $v_{\mathrm{c}}$ redwards of the optical/NIR regime, while the obtained optical/NIR data for GRBs 090323 and 090902B very probably probe the spectral region of $v_{\mathrm{m}}<v_{\mathrm{opt}}<v_{\mathrm{c}}$.

\subsection{Bumps in the afterglow light curves}

Variability superimposed on to a power-law decay as seen in GRBs 090323 and 090328 (Figs. 5 and 7) is frequently observed in well sampled optical afterglow light curves (e.g., Lazzati et al. 2002; Greiner et al. 2003; Lipkin et al. 2004; Updike et al. 2008; Perley et al. 2008; Krühler et al. 2009b). Given the low amplitude, smooth structure, time, and roughly equal color to the power-law component, the observed variability in the light curve of GRB 090323 is most likely related to a process intrinsic to the generic forward shock or the circumburst medium, and not to late inner engine activity. The most commonly inferred mechanism for producing variability as observed in the optical afterglow of GRB 090323 is additional energy injected into the afterglow via refreshed shocks (Rees \& Meszaros 1998), although an internal origin, i.e. an optical flare related to late central engine activity can not be ruled out. In GRB 090328 there is minor evidence for a slightly redder spectra in the bump component peaking at $\sim 350 \mathrm{ks}$ post burst, which would support a different origin of the observed variability than forward shock emission. Given the uncertainties in the measurement however, the spectral index is consistent with the afterglow slope at the $2 \sigma$ level.

Various models are emerging which explain delayed $\mathrm{GeV}$ with respect to the $\mathrm{keV}-\mathrm{MeV}$ emission in Fermi bursts, among others an external shock model (e.g. Ghisellini et al. 2010; Kumar \& Barniol Duran 2009b) or a hadronic model (Razzaque et al. 2009). Razzaque et al. (2009) demonstrate that synchrotron radiation from cosmic-ray protons accelerated in a GRB, delayed by the proton synchrotron cooling timescale in a jet of magnetically-dominated shocked plasma moving at highly relativistic speeds, explains the delayed $\mathrm{GeV}$ emission as observed in many Fermi/LAT bursts. A second generation prompt electron synchrotron component from attenuated proton synchrotron radiation makes enhanced soft $\mathrm{X}$-ray to $\mathrm{MeV}$ gamma-ray emission, which is, however, too weak to be detected. In this scenario the implicit assumption is made that the outflow impacts either a previously ejected shell or a pre-existing uniform density medium with an extent of $r \sim 6 \times 10^{16}(\Gamma / 1000)^{2}(\delta t / 10 \mathrm{~s}) \mathrm{cm}$. For a 3 s delay in GRB $090323 / 28$ this corresponds to a light travel time of 8-10 days, surprisingly similar to the timing of the bump in the optical/NIR light curve as observed with GROND. It is conceivable that the delayed $\mathrm{GeV}$ emission and the late, likely achromatic bumps in the afterglow light curve of GRBs could be related. One possible scenario would be Compton scattering of this proton synchrotron emission at the shell material, thus creating an achromatic bump with a power-law of photon index -2 (or even steeper) depending on the efficiency of the scattering. We note however, that density enhancements in the circumburst medium alone can not account for significant rebrightnings or strong bumps observed in optical afterglow light curves (Nakar \& Granot 2007). To reproduce the observed variability in optical afterglow light curves, a second process is required, possibly the variation of the microphysical shock parameters along the shock condition as suggested by Kong et al. (2010). 


\section{Conclusion}

We have presented the multi-colour afterglow observations of four bursts with high energy emission including three long and one short burst. In addition, we present spectroscopic observations and redshift determinations of two of these bursts. It is now possible for the first time to combine the high energy information from the prompt emission with the afterglow, host galaxy and redshifts of these sources. Follow-up of GRBs detected by Fermi with the seven band imager, GROND, allows simultaneous determination of the temporal, $\alpha$, and spectral, $\beta_{\text {opt }}$, indices of the burst afterglows. The long bursts exhibit power-law decay indices $(\alpha)$ from less than 1 to $\sim 2.3$ and spectral indices $\left(\beta_{\text {opt }}\right)$ from 0.65 to $\sim 1.2$ which are fairly standard for GRB afterglows. Moreover, an estimation of the jet break time is vital to determine the broadband properties and energetics of these events. The redshifts of the long bursts span the range from 0.7354 to 3.57 and the beaming corrected energies differ by a factor of at least $\sim 30$ with a value of $E_{\gamma} \gtrsim 2.2 \times 10^{52} \mathrm{erg}$ for GRB 090902B and are not compatible with a standard candle. Interestingly, the higher redshift bursts detected by the LAT are very luminous in the $\mathrm{keV}-\mathrm{MeV}$ region of the prompt spectrum, have high $E_{\gamma, \text { iso }}$ and $E_{\gamma}$ and also exceptionally bright afterglows. The lower redshift GRB 090328 is the exception in this respect, with a more standard $E_{\gamma \text {,iso }}$ as compared to Swift GRBs. The host galaxies of GRB 090328 and GRB 090510 fit well within the distributions of the long and short burst host galaxies respectively. Future photometric and spectroscopic follow-up of these rare Fermi bursts with high-energy emission is crucial to further investigate the energetics and host galaxies of a larger sample.

Acknowledgements. Based on observations made with ESO Telescopes at the Paranal Observatories under programme ID 083.D-0903 and 283.D-5059. We thank the staff at the European Southern Observatory for the generous allocation of observing time and execution of observations. In particular we thank Tim de Zeeuw, Andreas Kaufer, Michael Sterzik, Cedric Ledoux, Thomas Rivinius, Chris Lidman, F. J. Selman and I. Condor. Part of the funding for GROND (both hardware as well as personnel) was generously granted from the Leibniz-Prize to Prof. G. Hasinger (DFG grant HA 1850/28-1). S.M.B. acknowledges support of a European Union Marie Curie European Reintegration Grant within the 7th Program under contract number PERG04-GA-2008-239176. T.K. acknowledges support by the DFG cluster of excellence Origin and Structure of the Universe. D.A.K. acknowledges support by the Thüringer Landessternwarte Tautenburg, and thanks S. Stecklum for observing time, as well as U. Laux and F. Ludwig for performing the observations. We thank the anonymous referee for comments which helped to improved the paper.

Note added in proof. We note the publication of three relevant papers since the submission of this paper: Pandey et al. (2010), Rau et al. (2010) and Cenko et al. (2010b).

\section{References}

Abazajian, K. N., Adelman-McCarthy, J. K., Agüeros, M. A., et al. 2009, ApJS, 182,543

Abdo, A. A., Ackermann, M., Ajello, M., et al. 2009a, Nature, 462, 331

Abdo, A. A., Ackermann, M., Ajello, M., et al. 2009b, ApJ, 706, L138

Abdo, A. A., Ackermann, M., Arimoto, M., et al. 2009c, Science, 323, 1688

Abdo, A. A., Ackermann, M., Asano, K., et al. 2009d, ApJ, 707, 580

Allen, B., Yock, P., de Ugarte Postigo, A., et al. 2009, GRB Coordinates Network, 9058

Amati, L., Frontera, F., \& Guidorzi, C. 2009, A\&A, 508, 173

Amati, L., Guidorzi, C., Frontera, F., et al. 2008, MNRAS, 391, 577

Amelino-Camelia, G., \& Smolin, L. 2009, Phys. Rev. D, 80, 084017

Amelino-Camelia, G., Ellis, J., Mavromatos, N. E., Nanopoulos, D. V., \& Sarkar, S. 1998, Nature, 393, 763

Antonelli, L. A., D’Avanzo, P., Perna, R., et al. 2009, A\&A, 507, L45

Appenzeller, I., Fricke, K., Fürtig, W., et al. 1998, The Messenger, 94, 1

Atwood, W. B., et al. 1994, Nuclear Instruments and Methods in Physics Research A, 342, 302

Atwood, W. B., Abdo, A. A., Ackermann, M., et al. 2009, ApJ, 697, 1071

Barthelmy, S. D., Barbier, L. M., Cummings, J. R., et al. 2005, Space Sci. Rev., 120,143
Beckwith, S. V. W., Stiavelli, M., Koekemoer, A. M., et al. 2006, AJ, 132, 1729 Berger, E. 2009, ApJ, 690, 231

Berger, E., Kulkarni, S. R., Pooley, G., et al. 2003, Nature, 426, 154 Berger, E., Price, P. A., Cenko, S. B., et al. 2005, Nature, 438, 988 Berger, E., Fox, D. B., Price, P. A., et al. 2007, ApJ, 664, 1000 Bertin, E., \& Arnouts, S. 1996, A\&AS, 117, 393

Bissaldi, E. 2009, GRB Coordinates Network, 9933

Bissaldi, E., \& Connaughton, V. 2009, GRB Coordinates Network, 9866 Bissaldi, E., et al. 2010 [arXiv: 1002 . 4194]

Bloom, J. S., Kulkarni, S. R., \& Djorgovski, S. G. 2002, AJ, 123, 1111 Bloom, J. S., Frail, D. A., \& Kulkarni, S. R. 2003, ApJ, 594, 674 Bloom, J. S., Prochaska, J. X., Pooley, D., et al. 2006, ApJ, 638, 354 Bolzonella, M., Miralles, J.-M., \& Pelló, R. 2000, A\&A, 363, 476 Bouchet, P., Lequeux, J., Maurice, E., Prevot, L., \& Prevot-Burnichon, M. L. 1985, A\&A, 149, 330

Bouvier, A., Band, D., Bregeon, J., et al. 2008, GRB Coordinates Network, 8183 Bruzual, G., \& Charlot, S. 2003, MNRAS, 344, 1000

Burrows, D. N., Hill, J. E., Nousek, J. A., et al. 2005, Space Sci. Rev., 120, 165

Butler, N. R., Kocevski, D., Bloom, J. S., \& Curtis, J. L. 2007, ApJ, 671, 656 Campana, S., Guidorzi, C., Tagliaferri, G., et al. 2007, A\&A, 472, 395 Cenko, S. B., Bloom, J. S., Morgan, A. N., \& Perley, D. A. 2009, GRB Coordinates Network, 9053

Cenko, S. B., Frail, D. A., Harrison, F. A., et al. 2010a, ApJ, 711, 641

Cenko, S. B., Frail, D. A., Harrison, F. A., et al. 2010b, ApJ, submitted [arXiv: 1004.2900$]$

Chandra, P., \& Frail, D. A. 2009, GRB Coordinates Network, 9889

Chevalier, R. A., \& Li, Z. 2000, ApJ, 536, 195

Chornock, R., Perley, D. A., Cenko, S. B., \& Bloom, J. S. 2009, GRB Coordinates Network, 9028

Christensen, L., Hjorth, J., \& Gorosabel, J. 2004, A\&A, 425, 913

Cucchiara, A., Fox, D. B., Tanvir, N., \& Berger, E. 2009, GRB Coordinates Network, 9873

Cutini, S., Vasileiou, V., \& Chiang, J. 2009, GRB Coordinates Network, 9077 D’Avanzo, P., Malesani, D., Covino, S., et al. 2009, A\&A, 498, 711 de Jager, O. C., \& Stecker, F. W. 2002, ApJ, 566, 738

de Palma, F., Bissaldi, E., Tajima, H., et al. 2009a, GRB Coordinates Network, 9872

de Palma, F., Bregeon, J., \& Tajima, H. 2009b, GRB Coordinates Network, 9867 De Pasquale, M., Schady, P., Kuin, N. P. M., et al. 2010, ApJ, 709, L146 Eichler, D., Livio, M., Piran, T., \& Schramm, D. N. 1989, Nature, 340, 126 Evans, P. A., Beardmore, A. P., Page, K. L., et al. 2009, MNRAS, 397, 1177 Finke, J. D., Razzaque, S., \& Dermer, C. D. 2010, ApJ, 712, 238 Fitzpatrick, E. L. 1986, AJ, 92, 1068

Fong, W., Berger, E., \& Fox, D. B. 2010, ApJ, 708, 9

Fox, D. B., Frail, D. A., Price, P. A., et al. 2005, Nature, 437, 845 Frail, D. A., Chandra, P., \& Cenko, B. 2009, GRB Coordinates Network, 9060 Frail, D. A., Kulkarni, S. R., Sari, R., et al. 2001, ApJ, 562, L55 Fruchter, A. S., Levan, A. J., Strolger, L., et al. 2006, Nature, 441, 463 Franceschini, A., Rodighiero, G., \& Vaccari, M. 2008, A\&A, 487, 837 Fryer, C. L., Woosley, S. E., \& Hartmann, D. H. 1999, ApJ, 526, 152 Fynbo, J. P. U., Jakobsson, P., Prochaska, J. X., et al. 2009, ApJS, 185, 526 Galama, T. J., Vreeswijk, P. M., van Paradijs, J., et al. 1998, Nature, 395, 670 Gehrels, N., Barthelmy, S. D., Burrows, D. N., et al. 2008, ApJ, 689, 1161 Gehrels, N., Chincarini, G., Giommi, P., et al. 2004, ApJ, 611, 1005 Gehrels, N., Sarazin, C. L., O’Brien, P. T., et al. 2005, Nature, 437, 851 Ghirlanda, G., Ghisellini, G., \& Lazzati, D. 2004, ApJ, 616, 331 Ghirlanda, G., Nava, L., Ghisellini, G., \& Firmani, C. 2007, A\&A, 466, 127 Ghisellini, G., Ghirlanda, G., Nava, L., \& Celotti, A. 2010, MNRAS, 403, 926 Giavalisco, M., Ferguson, H. C., Koekemoer, A. M., et al. 2004, ApJ, 600, L93 Giuliani, A., Fuschino, F., Vianello, G., et al. 2010, ApJ, 708, L84 Giuliani, A., Mereghetti, S., Fornari, F., et al. 2008, A\&A, 491, L25 Golenetskii, S., Aptekar, R., Mazets, S., et al. 2009, GRB Coordinates Network, 9344

González, M. M., Dingus, B. L., Kaneko, Y., et al. 2003, Nature, 424, 749 Gorosabel, J., Castro-Tirado, A. J., Guziy, S., et al. 2006, A\&A, 450, 87 Graham, J. F., Fruchter, A. S., Levan, A. J., et al. 2009, ApJ, 698, 1620 Greiner, J., Klose, S., Reinsch, K., et al. 2003, Nature, 426, 157 Greiner, J., Bornemann, W., Clemens, C., et al. 2008, PASP, 120, 405 Greiner, J., Clemens, C., Krühler, T., et al. 2009a, A\&A, 498, 89 Greiner, J., Krühler, T., McBreen, S., et al. 2009b, ApJ, 693, 1912 Grupe, D., \& Hoversten, E. 2009, GRB Coordinates Network, 9341 Guidorzi, C., Tanvir, N. R., Cano, Z., et al. 2009, GRB Coordinates Network, 9875

Guiriec, S., Connaughton, V., \& Briggs, M. 2009, GRB Coordinates Network, 9336

Hamuy, M., Walker, A. R., Suntzeff, N. B., et al. 1992, PASP, 104, 533

Hanlon, L. O., Bennett, K., Collmar, W., et al. 1994, A\&A, 285, 161 
Harrison, F., Cenko, B., Frail, D. A., Chandra, P., \& Kulkarni, S. 2009, GRB Coordinates Network, 9043

Hjorth, J., Sollerman, J., Møller, P., et al. 2003, Nature, 423, 847

Hjorth, J., Watson, D., Fynbo, J. P. U., et al. 2005, Nature, 437, 859

Hoversten, E. A., Barthelmy, S. D., Burrows, D. N., et al. 2009, GRB Coordinates Network, 9331

Huang, S., \& Gu, Q. 2009, MNRAS, 398, 1651

Hurley, K. 1992, in AIP, ed. W. S. Paciesas, \& G. J. Fishman, 265, 3

Hurley, K., Dingus, B. L., Mukherjee, R., et al. 1995, Nature, 374, 94

Hurley, K., Goldsten, J., von Kienlin, A., et al. 2009, GRB Coordinates Network, 9023, 1

Kaneko, Y., González, M. M., Preece, R. D., Dingus, B. L., \& Briggs, M. S. 2008, ApJ, 677, 1168

Kann, D. A., Klose, S., \& Zeh, A. 2006, ApJ, 641, 993

Kann, D. A., Klose, S., Zhang, B., et al. 2008 [arXiv:0804 . 1959]

Kann, D. A., Klose, S., Zhang, B., et al. 2009a [arXiv:0712.2186v2]

Kann, D. A., Laux, U., Ludwig, F., \& Stecklum, S. 2009b, GRB Coordinates Network, 9063

Kann, D. A., Laux, U., \& Stecklum, S. 2009c, GRB Coordinates Network, 9033 Kann, D. A., Laux, U., \& Stecklum, S. 2009d, GRB Coordinates Network, 9041

Kashlinsky, A., Arendt, R. G., Mather, J., \& Moseley, S. H. 2005, Nature, 438, 45

Kennea, J. 2009, GRB Coordinates Network, 9045

Kennea, J., \& Stratta, G. 2009, GRB Coordinates Network, 9868

Kennea, J., Evans, P., \& Goad, M. 2009a, GRB Coordinates Network, 9024

Kennea, J., Evans, P., \& Goad, M. 2009b, GRB Coordinates Network, 9046

Kneiske, T. M., Bretz, T., Mannheim, K., \& Hartmann, D. H. 2004, A\&A, 413, 807

Kong, S. W., Wong, A. Y. L., Huang, Y. F., \& Cheng, K. S. 2010, MNRAS, 402, 409

Kouveliotou, C., Meegan, C. A., Fishman, G. J., et al. 1993, ApJ, 413, L101

Krühler, T., Küpcü Yoldaş, A., Greiner, J., et al. 2008, ApJ, 685, 376

Krühler, T., Greiner, J., Afonso, P., et al. 2009a, A\&A, 508, 593

Krühler, T., Greiner, J., McBreen, S., et al. 2009b, ApJ, 697, 758

Kuin, N. P. M., \& Hoversten, E. A. 2009, GRB Coordinates Network, 9342

Kuin, N. P. M., Oates, S., de Pasquale, M., Hoversten, E. A., \& Marshall, F. 2009, GRB Coordinates Network, 9351

Kumar, P., \& Barniol Duran, R. 2009a [arXiv: 0910. 5726]

Kumar, P., \& Barniol Duran, R. 2009b, MNRAS, 400, L75

Lazzati, D., Rossi, E., Covino, S., Ghisellini, G., \& Malesani, D. 2002, A\&A, 396, L5

Lee, W. H., \& Ramirez-Ruiz, E. 2007, New J. Phys., 9, 17

Levan, A. J., Tanvir, N. R., Fruchter, A. S., et al. 2006a, ApJ, 648, L9

Levan, A. J., Wynn, G. A., Chapman, R., et al. 2006b, MNRAS, 368, L1

Levan, A. J., Jakobsson, P., Hurkett, C., et al. 2007, MNRAS, 378, 1439

Levesque, E. M., Bloom, J. S., Butler, N. R., et al. 2010, MNRAS, 401, 963

Lipkin, Y. M., Ofek, E. O., Gal-Yam, A., et al. 2004, ApJ, 606, 381

Longo, F., Moretti, E., Barbiellini, G., et al. 2009, GRB Coordinates Network, 9343

Malesani, D., Tagliaferri, G., Chincarini, G., et al. 2004, ApJ, 609, L5

Malesani, D., Goldoni, P., Fynbo, J., et al. 2009, GRB Coordinates Network, 9942

Marshall, F. E., \& Hoversten, E. A. 2009, GRB Coordinates Network, 9332

Mattingly, D. 2005, Living Reviews in Relativity, 8, 5

Mazets, E. P., Golenetskii, S. V., Ilyinskii, V. N., et al. 1981, Ap\&SS, 80, 119

McEnery, J., Chiang, J., \& Hanabata, Y. 2009a, GCN Circular, 9985

McEnery, J., Cutini, S., Ohno, M., Koerding, E., \& Connaughton, V. 2009b, GRB Coordinates Network, 9044

McEnery, J., Chiang, J., Omodei, N., \& Nakamori, T. 2010, GCN Circular, 10333

Meegan, C., Lichti, G., Bhat, P. N., et al. 2009, ApJ, 702, 791

Mészáros, P. 2006, Rep. Prog. Phys., 69, 2259

Metcalfe, L., Kneib, J., McBreen, B., et al. 2003, A\&A, 407, 791

Michelson, P. F. 1996, in SPIE Conf. 2806, ed. B. D. Ramsey, \& T. A. Parnell, 31

Nakar, E. 2007, Phys. Rep., 442, 166

Nakar, E., \& Granot, J. 2007, MNRAS, 380, 1744

Norris, J. P., Cline, T. L., Desai, U. D., et al. 1984, Nature, 308, 434

Nousek, J. A., Kouveliotou, C., Grupe, D., et al. 2006, ApJ, 642, 389

Nysewander, M., Fruchter, A. S., \& Pe'er, A. 2009, ApJ, 701, 824

Oates, S. R. 2009, GRB Coordinates Network, 9048

Ohmori, N., Noda, K., Sonoda, E., et al. 2009, GRB Coordinates Network, 9355

Ohno, M., \& Pelassa, V. 2009, GRB Coordinates Network, 9334

Ohno, M., McEnery, J., \& Pelassa, V. 2008, GRB Coordinates Network, 8903

Ohno, M., Cutini, S., McEnery, J., Chiang, J., \& Koerding, E. 2009, GRB Coordinates Network, 9021

Olivares, F., Afonso, F., Greiner, J., et al. 2009a, GRB Coordinates Network, 9874
Olivares, F., Klose, S., Krühler, T., \& Greiner, J. 2009b, GRB Coordinates Network, 9352

Olofsson, G., Ergon, M., Malesani, D., et al. 2009, GRB Coordinates Network, 9338, 1

Omodei, N. 2008, GRB Coordinates Network, 8407

Omodei, N., Granot, J., Meszaros, P., et al. 2009, GRB Coordinates Network, 9350

Palma, F. d., Bari, I., Omodei, N., McEnery, J., \& Vasileiou, V. 2009, GCN Circular, 10163

Panaitescu, A. 2005, MNRAS, 362, 921

Panaitescu, A., Mészáros, P., Gehrels, N., Burrows, D., \& Nousek, J. 2006, MNRAS, 366, 1357

Pandey, S. B., Zheng, W., Yuan, F., \& Akerlof, C. 2009, GRB Coordinates Network, 9878

Pandey, S. B., Swenson, C. A., Perley, D. A., et al. 2010, ApJ, 714, 799

Perley, D. A., Bloom, J. S., Butler, N. R., et al. 2008, ApJ, 672, 449

Perley, D., Kleiser, I. K. W., \& Rex, J. M. 2009a, GRB Coordinates Network, 9870

Perley, D. A., Cenko, S. B., Bloom, J. S., et al. 2009b, AJ, 138, 1690

Perri, M., \& Stratta, G. 2009, GRB Coordinates Network, 9031

Piran, T. 2004, Rev. Mod. Phys., 76, 1143

Piron, F., Longo, F., Iafrate, G., et al. 2009, GRB Coordinates Network, 9584

Polletta, M., Tajer, M., Maraschi, L., et al. 2007, ApJ, 663, 81

Prochaska, J. X., Bloom, J. S., Chen, H.-W., et al. 2006, ApJ, 642, 989

Racusin, J. L., Karpov, S. V., Sokolowski, M., et al. 2008, Nature, 455, 183

Racusin, J. L., Liang, E. W., Burrows, D. N., et al. 2009, ApJ, 698, 43

Rau, A., Connaughton, V., \& Briggs, M. 2009a, GRB Coordinates Network, 9057

Rau, A., McBreen, S., Krühler, T., \& Greiner, J. 2009b, GRB Coordinates Network, 9353

Rau, A., Savaglio, S., Krühler, T., et al. 2010, ApJ, [arXiv: 1004.3261]

Razzaque, S., Dermer, C. D., \& Finke, J. D. 2009 [arXiv: 0908. 0513]

Rees, M. J., \& Meszaros, P. 1998, ApJ, 496, L1

Roming, P. W. A., Kennedy, T. E., Mason, K. O., et al. 2005, Space Sci. Rev., 120,95

Rossi, A., de Ugarte Postigo, A., Ferrero, P., et al. 2008, A\&A, 491, L29

Salvaterra, R., Della Valle, M., Campana, S., et al. 2009, Nature, 461, 1258

Sari, R., Piran, T., \& Narayan, R. 1998, ApJ, 497, L17

Sari, R., Piran, T., \& Halpern, J. P. 1999, ApJ, 519, L17

Savage, B. D., \& Sembach, K. R. 1991, ApJ, 379, 245

Savaglio, S., Glazebrook, K., \& LeBorgne, D. 2009, ApJ, 691, 182

Scargle, J. D., Norris, J. P., \& Bonnell, J. T. 2008, ApJ, 673, 972

Schlegel, D. J., Finkbeiner, D. P., \& Davis, M. 1998, ApJ, 500, 525

Seaton, M. J. 1979, MNRAS, 187, 73P

Skrutskie, M. F., Cutri, R. M., Stiening, R., et al. 2006, AJ, 131, 1163

Spitzer, L. 1978, Physical processes in the interstellar medium (New York: Wiley)

Stecker, F. W., Malkan, M. A., \& Scully, S. T. 2006, ApJ, 648, 774

Stratta, G., D'Elia, V., \& Perri, M. 2009, GRB Coordinates Network, 9876

Swenson, C. A., \& Siegel, M. H. 2009, GRB Coordinates Network, 9869

Swenson, C. A., \& Stratta, G. 2009, GRB Coordinates Network, 9877

Tanvir, N. R., Fox, D. B., Levan, A. J., et al. 2009, Nature, 461, 1254

Tinney, C., Stathakis, R., Cannon, R., \& Galama, T. 1998, IAU Circ., 6896, 3

Tody, D. 1993, in Astronomical Data Analysis Software and Systems II, ed. R. J. Hanisch, R. J. V. Brissenden, \& J. Barnes, ASP Conf. Ser., 52,

Troja, E., King, A. R., O’Brien, P. T., Lyons, N., \& Cusumano, G. 2008, MNRAS, 385, L10

Uehara, T., Takahashi, H., \& McEnery, J. 2009, GRB Coordinates Network, 9934

Updike, A. C., Haislip, J. B., Nysewander, M. C., et al. 2008, ApJ, 685, 361

Updike, A., Klose, S., Clemens, C., \& Greiner, J. 2009a, GRB Coordinates Network, 9054

Updike, A. C., Filgas, R., Krühler, T., Greiner, J., \& McBreen, S. 2009b, GRB Coordinates Network, 9026

van der Horst, A. J. 2009, GRB Coordinates Network, 9047

van der Horst , A. J., Kamble, A. P., Wijers, R. A. M. J., \& Kouveliotou, C. 2009, GRB Coordinates Network, 9883

Vedrenne, G., \& Atteia, J. L. 2009, Gamma-ray Bursts: The brightest explosions in the Universe (Springer Praxis Books), Astronomy and Planetary Sciences Jointly published with Praxis Publishing

Willmer, C. N. A., Faber, S. M., Koo, D. C., et al. 2006, ApJ, 647, 853

Wilson-Hodge, C., Connaughton, V., Longo, F., \& Omodei, N. 2008, GRB Coordinates Network, 8723

Zeh, A., Klose, S., \& Hartmann, D. H. 2004, ApJ, 609, 952

Zeh, A., Klose, S., \& Kann, D. A. 2006, ApJ, 637, 889

Zhang, B., \& Mészáros, P. 2004, Int. J. Mod. Phys. A, 19, 2385

Zhang, B., Fan, Y. Z., Dyks, J., et al. 2006, ApJ, 642, 354

Zhang, B., Zhang, B., Virgili, F. J., et al. 2009, ApJ, 703, 1696 